\title{
Broadband longwave radio remote sensing instrumentation
}

Cite as: Rev. Sci. Instrum. 89, 094501 (2018); https://doi.org/10.1063/1.5041419

Submitted: 24 May 2018 . Accepted: 17 August 2018 . Published Online: 07 September 2018

Morris B. Cohen (D), Ryan K. Said, Evans W. Paschal, Jackson C. McCormick (D), Nicholas C. Gross (D), Lee Thompson, Marc Higginson-Rollins, Umran S. Inan, and Jeffrey Chang

\section{ARTICLES YOU MAY BE INTERESTED IN}

An open and flexible digital phase-locked loop for optical metrology

Review of Scientific Instruments 89, 093103 (2018); https://doi.org/10.1063/1.5039344

A novel, tunable, multimodal microwave system for microwave reflectometry system Review of Scientific Instruments 89, 093501 (2018); https://doi.org/10.1063/1.5033968

Metrological large range magnetic force microscopy

Review of Scientific Instruments 89, 093703 (2018); https://doi.org/10.1063/1.5035175

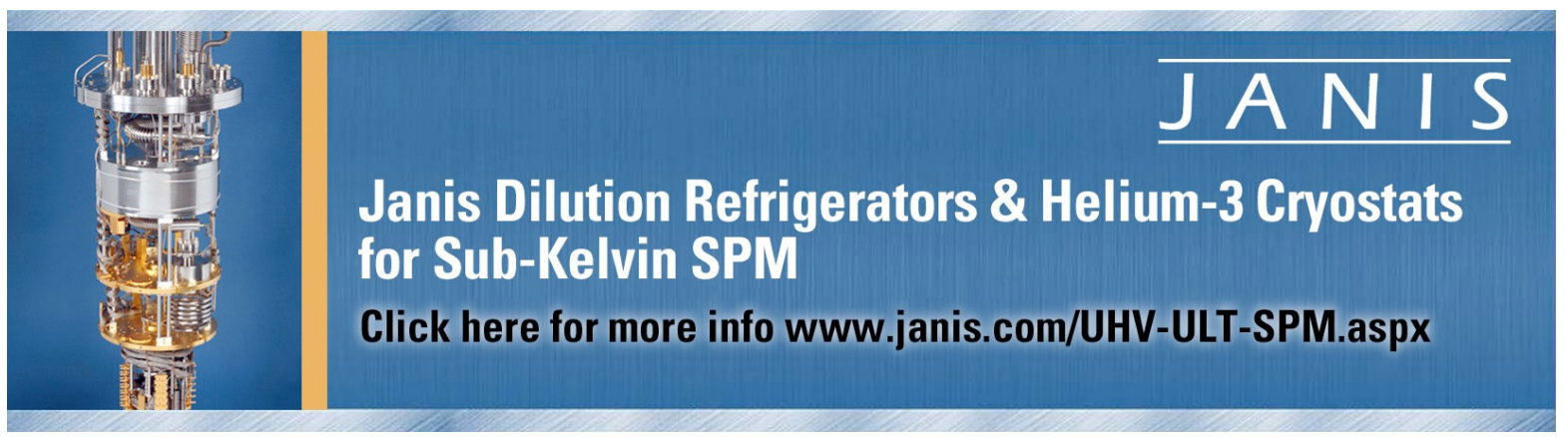




\title{
Broadband longwave radio remote sensing instrumentation
}

\author{
Morris B. Cohen, ${ }^{1,2, a)}$ Ryan K. Said, ${ }^{3}$ Evans W. Paschal, ${ }^{4}$ Jackson C. McCormick, ${ }^{1}$ Nicholas \\ C. Gross, ${ }^{1}$ Lee Thompson, ${ }^{5}$ Marc Higginson-Rollins, ${ }^{1}$ Umran S. Inan, ${ }^{6,7}$ and Jeffrey Chang ${ }^{8}$ \\ ${ }^{1}$ School of Electrical and Computer Engineering, Georgia Institute of Technology, Atlanta, \\ Georgia 30332-0360, USA \\ ${ }^{2}$ Center for Space Technology and Research, Georgia Institute of Technology, Atlanta, \\ Georgia 30332-0360, USA \\ ${ }^{3}$ Vaisala, Inc., Boulder Operations, Louisville, Colorado 80027, USA \\ ${ }^{4}$ Whistler Radio Services, Anderson Island, Washington 98303, USA \\ ${ }^{5}$ SpaceX, Inc., Hawthorne, California 90250, USA \\ ${ }^{6}$ Department of Electrical Engineering, Stanford University, Stanford, California 94305, USA \\ ${ }^{7}$ Office of the Rector, Koc University, Sariyer, Istanbul, Turkey \\ ${ }^{8}$ Omnicell, Inc., Mountain View, California 94043, USA
}

(Received 24 May 2018; accepted 17 August 2018; published online 7 September 2018)

\begin{abstract}
We present the performance characteristics of a high-sensitivity radio receiver for the frequency band 0.5-470 kHz, known as the Low Frequency Atmospheric Weather Electromagnetic System for Observation, Modeling, and Education, or LF AWESOME. The receiver is an upgraded version of the VLF AWESOME, completed in 2004, which provided high sensitivity broadband radio measurements of natural lightning emissions, transmitting beacons, and radio emissions from the near-Earth space environment. It has been deployed at many locations worldwide and used as the basis for dozens of scientific studies. We present here a significant upgrade to the AWESOME, in which the frequency range has been extended to include the LF and part of the medium frequency (MF) bands, the sensitivity improved by $10-25 \mathrm{~dB}$ to be as low as $0.03 \mathrm{fT} / \sqrt{\mathrm{Hz}}$, depending on the frequency, and timing error reduced to $15-20 \mathrm{~ns}$ range. The expanded capabilities allow detection of radio atmospherics from lightning strokes at global distances and multiple traverses around the world. It also allows monitoring of transmitting beacons in the LF/MF band at thousands of $\mathrm{km}$ distance. We detail the specification of the LF AWESOME and demonstrate a number of scientific applications. We also describe and characterize a new algorithm for minimum shift keying demodulation for VLF/LF transmitters for ionospheric remote sensing applications. Published by AIP Publishing. https://doi.org/10.1063/1.5041419
\end{abstract}

\section{INTRODUCTION}

The low frequency radio band has long been of interest to both scientists and engineers. After more than a decade of effort to slowly increase the range of radio communications, the very first use of long distance (transatlantic) radio propagation was achieved by the famous spark gap experiment by Guglielmo Marconi in 1901, ${ }^{1}$ in which the morse code for the letter "s" was sent from Cornwall, England, to St. John's, Newfoundland. Proving his detractors wrong, Marconi correctly predicted that the signals would be guided around the Earth. But his explanation that the Earth itself would guide the wave was incorrect: The explanation for why it worked did not come until Oliver Heaviside and Arthur Kennelly proposed (in 1902) and then Appleton demonstrated (in 1923) ${ }^{2,3}$ the existence of an ionized layer at the top of the atmosphere, known as the ionosphere, which essentially forms the border between the neutral atmosphere and the space plasma environment, beginning at $90 \mathrm{~km}$ altitude to form what is now known as the E region. Marconi's transatlantic reception was done with a radio frequency in the $100 \mathrm{~s}$ of $\mathrm{kHz}$ range, which reflected from the ionosphere.

a)mcohen@gatech.edu. URL: http://LF.gatech.edu.
This discovery gave rise in the ensuing decades to the field of long wave radio science, which we roughly define here as the use of radio waves below $1 \mathrm{MHz}$. Longwave radio science was and is a fantastically productive means by which we study the natural electricity of the Earth and its surrounding space environment. Most radio wave energy below $1 \mathrm{MHz}$ reflects from the ionosphere, thereby interacting with this highly variable region. Some of the energy that does escape into space engenders its own dizzying set of physical phenomena. Entire books are filled with the rich array of scientific discoveries that resulted from long wave science. We have no hope of capturing all of that here, but we list a few of the most notable in the spirit of declaring our motivations. Good reviews of extremely low frequency (ELF)/VLF radio wave science can be found in Refs. 4-6.

Well before we had the means to broadcast radio, lightning was already pumping its energy into electromagnetic waves. Lightning is perhaps the most powerful physical process on the Earth, demonstrated not only by its awe-inspiring sight and destructive ability but by its unseen physical processes like $\gamma$-ray production rival collapsing stars. ${ }^{7}$ Although only $0.01 \%-1 \%$ of lightning's energy is released electrically at the strike point (Ref. 8, p. 12), rather than through thunder and heating, this is enough to have dramatic effects. The bulk of lightning's electromagnetic energy is radiated between 1 and 
$100 \mathrm{kHz}$ (Ref. 9, chap. 3), and due to reflection from the lowest part of the ionosphere, impulsive bits of radiation called "sferics" can be observed many thousands of $\mathrm{km}$ away from the source. ${ }^{10}$ Recently, this result has been leveraged to geolocate and characterize the vast majority $(80 \%)$ of cloud-to-ground (CG) lightning flashes, and a significant fraction (30\%) of intracloud (IC) lightning flashes, ${ }^{11}$ which occur about $3.5 \times 10^{6}$ times per day on average. ${ }^{12}$ The electromagnetic pulse released from lightning impacts the ionosphere producing an optical emission known as an "elve," 13 which is one of several mechanisms by which lightning interacts with the ionosphere. Perhaps most interesting is the existence of "sprites," which were first theorized many decades ago ${ }^{14}$ and discovered accidentally many decades later. ${ }^{15}$ Sprites have since been studied and observed in many different regions around the world. 5,16-22 A rarer but perhaps more bizarre phenomenon known as gigantic jets ${ }^{23,24}$ has also followed suit. A small fraction of the lightning electromagnetic pulse radiation (EMP) also propagates through the ionosphere ${ }^{25}$ and into the space environment, triggering electron precipitation from the radiation belts. ${ }^{26,27}$

Long before the advent of GPS, a network of VLF transmitters formed the first global navigation system, via the United States "Omega" system, ${ }^{28}$ which consisted of a number of beacons operating between 10 and $14 \mathrm{kHz}$ around the globe. Via phase multilateration and regional/diurnal ionospheric phase correction tables, an accuracy of 1-2 nmi became possible. Russia continues to operate a similar system, with a more limited coverage area, known as "Alpha."

VLF has for many decades been a fundamental component of submarine communication, with carefully engineered systems both for the transmitter ${ }^{29,30}$ and the receiver ${ }^{31}$ leverating the propagation to global distances and penetrating $10 \mathrm{~s}$ of $\mathrm{m}$ into seawater via the skin effect. These signals, once established for submarine communications, have subsequently proven scientifically valuable as a diagnostic for the lower ionosphere $(60-90 \mathrm{~km}),{ }^{32,33}$ which is otherwise too high for balloons, too low for satellites, and not sufficiently ionized for radars. ${ }^{34}$ VLF transmitters are generally large facilities covering hundreds to thousands of acres, are expensive to construct, and have very limited bandwidth. Some alternatives have been the subject of research, including many efforts on ELF/VLF generation via nonlinear modulation of the D region conductivity with high power HF waves. ${ }^{35-39}$

Lower ionospheric disturbances result from a diverse array of sources, which make simple passive monitoring of the VLF/LF band incredibly powerful as a geophysical tool. The geophysical phenomena that impact the lower ionosphere in measurable ways include solar flares, ${ }^{40-45}$ general solar x-ray activity, ${ }^{46}$ solar proton events, ${ }^{47,48}$ solar eclipses, ${ }^{49-59}$ solar zenith angle, ${ }^{32,60}$ astronomical gamma-ray bursts and magnetars, ${ }^{61-65}$ auroral precipitation, ${ }^{66,67}$ electron precipitation from geomagnetic storms ${ }^{68,69}$ including around the south Atlantic anomaly, ${ }^{70,71}$ sprite halos, ${ }^{72,73}$ direct heating from lightning, ${ }^{74-76}$ direct heating from VLF transmitters, ${ }^{77,78}$ electron precipitation induced by lightning, ${ }^{26,79,80}$ electron precipitation triggered by VLF transmitters, ${ }^{81,82}$ gravity waves, ${ }^{83-86}$ earthquakes and seismic activity, ${ }^{87-90}$ changes in mesopause temperatures, ${ }^{91}$ magnetosphere-atmosphere coupling, ${ }^{92}$ seasonal variations, ${ }^{93}$ solar cycle variations, ${ }^{94,95}$ meteorology, ${ }^{96}$ and lunar tides, ${ }^{97}$ among others. As a result, the field of VLF remote sensing has evolved as a primary means to study the $D$-region's response to a host of geophysical phenomena. There has even been some discussion of a $C$ region of the ionosphere. $^{98}$ The VLF transmitter energy also escapes into the magnetosphere ${ }^{99-101}$ where it may also impact radiation belt dynamics. ${ }^{102}$ A recent review of many of these topics was presented in Ref. 6.

The natural VLF/LF energy radiated from lightning is also a useful ionospheric diagnostic. The authors of Ref. 103 observed interference on submarine communications cables, and noted the presence of "tweeks" lasting one-eighth of a second, along with diurnal variations. Tweeks arise at frequencies close to the first-order waveguide cutoff $(\sim 1.8 \mathrm{kHz})$, where the group velocity is slower, so the energy is elongated in time. The authors of Ref. 49 showed variations in the frequency and amplitude of tweeks during a solar eclipse. This line of research has been continued by several others since then. ${ }^{58,104}$ Reference 105 utilized the full waveform of sferics for remote sensing of the ionosphere and found good agreement between the mode theory of VLF propagation and observed waveforms, which was further evolved in Ref. 106. Reference 107 added access to precise lightning geolocation data and was then able to select $D$-region waveguide properties for long propagation paths based on sferic amplitude spectra. Reference 108 augmented this approach by utilizing the time-domain waveform and including the LF content, which is particularly useful for medium range (hundreds of $\mathrm{km}$ ) propagation distances. Reference 109 utilized this technique to detect a reduction in nighttime ionospheric electron density from thunderstorms.

The Low Frequency (LF, 30-300 kHz) and Medium Frequency (MF, 300-3000 kHz) bands have also been exploited for navigation and communications. Although the Omega navigation system was decommissioned in 1997, the LORAN-C system continued operation around $100 \mathrm{kHz},{ }^{110}$ although it too largely shut down in 2011. The LF/MF band is also used to broadcast ionospheric corrections in the US via the Nationwide Differential GPS (NDGPS) network.

As the scientific and engineering applications of VLF/LF waves have grown, receivers have evolved, as well. Most radio receivers in this band utilize either a monopole whip or a magnetic loop antenna. A prominent recent example of an electric field receiver sampled at $1 \mathrm{MHz}$ is described in Ref. 111. A prominent ELF receiver (extending down to a fraction of a $\mathrm{Hz}$ ) is described in Ref. 112.

Magnetic field sensors are generally found in two varieties: air core and ferrite core. Satellite-based magnetic field receivers usually use ferrite core coils due to their compact size. ${ }^{113,114}$ But ferrite coils struggle with resonances that limit the frequency response. For example, a review in Ref. 115 discussed the issue of resonances, presented techniques to optimize the design, and showed example designs for ferrite core loops in which the sensitivity achieved is $0.1-1.0 \mathrm{fT} / \sqrt{ } \mathrm{Hz}$. In addition, ferrite materials have stability issues that make their practical properties in general different from the material properties. $^{116}$

Consequently, air-core loop antennas may be better than ferrite-core antennas when size is not a major constraint, as they deliver better sensitivity and more linearity. But capturing 
this sensitivity after amplification and digitization is not easy since it requires a low-noise and low-impedance amplifier. ${ }^{117}$

It is worth mentioning a third technology that is actively being investigated, whose derivatives hold significant promise for high sensitivity at chip-scale sizes, known as a superconducting quantum interference device, ${ }^{118,119}$ but in practice these have so far been difficult to work with and not very rugged and require cooling a superconductor. The preamplifier design also faces stability issues ${ }^{120}$ due to the low impedance of SQUIDs. Even so, the sensitivity of advanced atomic magnetometers is still in the range of $1 \mathrm{pT} / \sqrt{ } \mathrm{Hz},{ }^{121}$ which on its own does not yet improve on traditional magnetic field receivers.

Over the past several decades, a high-sensitivity VLF receiver using conventional magnetic field induction was developed at Stanford University and utilized in a variety of geophysical experiments. The receiver utilizes air-core loop antennas and a transformer for impedance matching to a low noise amplifier (LNA). The antenna and LNA matching design is described in Refs. 122 and 123. The most recent iteration of the design at Stanford was known as the "Atmospheric Weather Electromagnetic System for Observation Modeling and Education" (AWESOME) and is described in Ref. 124.

The AWESOME receiver has also been used to set up an international network of scientists largely in developing countries under the auspices of the NASA-sponsored International Heliophysical Year ${ }^{125,126}$ and forms the hardware basis of the GLD360 global lightning location network. ${ }^{11,127}$ In recent years, there has been an effort to adapt the VLF AWESOME to begin capturing the LF and part of the MF bands, and in the process, we also improved the sensitivity and resolved a timing accuracy issue that had limited the VLF AWESOME. This effort began at Stanford University but was completed at the Georgia Institute of Technology. In this paper, we describe this newly upgraded receiver and give its performance characteristics. We also give a detailed description of the minimum shift keying (MSK) demodulation algorithm used to monitor the amplitudes and phases of VLF transmitters for ionospheric remote sensing.

\section{RECEIVER DESCRIPTION}

The main science goals of the receiver are as follows:

- Sensitivity below $1 \mathrm{fT} / \sqrt{ } \mathrm{Hz}$ over the frequency range $2-450 \mathrm{kHz}$ to enable detection of weak few kA lightninggenerated impulses and $>50 \mathrm{~kW}$ VLF/LF/MF transmitter signals, at a continental to global range.

- Absolute timing accuracy better than 20 ns to enable $0.2^{\circ}$ of phase accuracy at $30 \mathrm{kHz}$, or no more than $6-\mathrm{m}$ of range error due to receiver timing jitter.

- Accurate magnetic field calibration to allow comparison with theoretical models.

We now proceed to describe the LF AWESOME receiver. The main components of the LF AWESOME are an aircore wire loop antenna, a preamplifier located nearby, a line receiver with GPS synchronization, an Analog-toDigital Converter (ADC), recording software, and a MSK

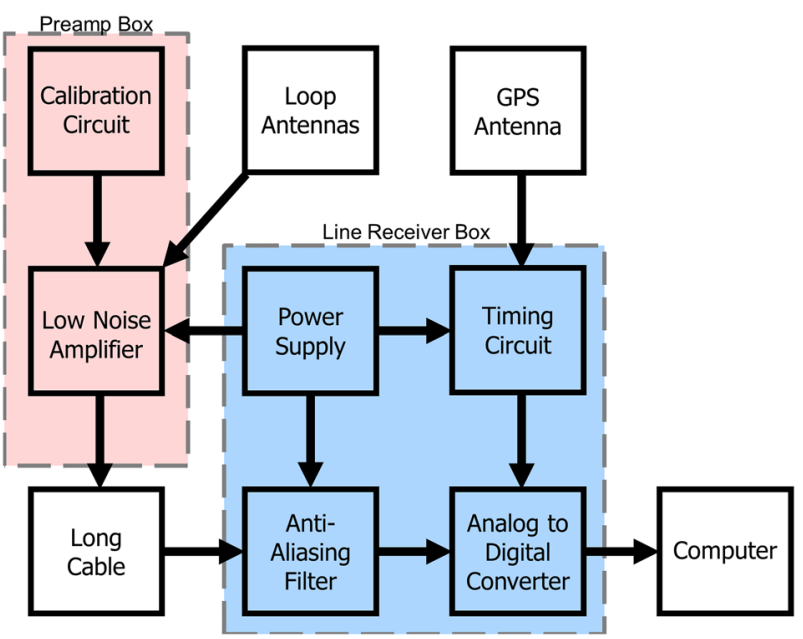

FIG. 1. Main system components of the LF AWESOME receiver.

demodulation algorithm. A block diagram of the system is shown in Fig. 1.

\section{A. System design}

The receiver design follows similar principles to those described in Ref. 124, which are briefly reviewed here. The LF AWESOME utilizes two orthogonal air-core loop antennas which sample, typically, the two components of the horizontal magnetic field. The receiver has capability for a third loop antenna, to record, for example, the vertical magnetic field, ${ }^{128}$ which may be more significant in the presence of scatterers such as power lines and buildings, but in the examples in this paper, we will ignore the vertical magnetic field component.

While VLF systems can also be designed with ferrite-core loops, air-core loops have a number of advantages, including lighter weight, less susceptibility to damage and degradation, ease of calibration, and better linearity. The air-core loop antenna produces an electromotive force $(V)$ in response to the magnetic flux density amplitude $(B)$ according to $V=\omega N A B$, where $N$ is the number of turns of wire in the loop and $A$ is the area of the antenna. This equation comes directly from Faraday's law of induction simplified by the fact that the magnetic field for a long-wavelength signal is very nearly constant across the loop area at any given moment in time. The resulting voltage is, however, quite small. For example, at $10 \mathrm{kHz}$, with a fairly large antenna, $25 \mathrm{~m}^{2}$ area and 5 turns, a $1 \mathrm{pT}$ magnetic flux density yields just $7.8 \mu \mathrm{V}$.

This voltage then flows through the impedance of an antenna and the electrical components connected to it to form a current, which can then be amplified. However, the antenna impedance consists of a resistance $R_{a}$ (defined by the finite conductivity of the wire) and an inductance $L_{a}$ (defined by the loop geometry and wire radius). The impedance of just the antenna is therefore dependent on the frequency, $R_{a}+j \omega L_{a}$, where $\omega$ is the angular frequency. Below a turnover frequency, defined by $\omega=\frac{R_{a}}{L_{a}}$, the antenna impedance is dominated by the resistance, whereas above this frequency, the impedance is dominated by the inductance. The strategy for achieving a flat frequency response, therefore, is to set the antenna turnover frequency below the frequencies of interest so that both the 
antenna impedance and the induced emf are proportional to frequency. This yields a net current that is roughly flat with frequency above the antenna turnover frequency.

To set a turnover frequency in the range of $100 \mathrm{~s}$ of $\mathrm{Hz}$, we want $R_{a}$ (in units of $\Omega$ ) to be roughly three orders of magnitude greater than $L_{a}$ (in units of $\mathrm{H}$ ). This still yields infinite possible combinations, though. In fact, as shown in Refs. 122 and 123 , the sensitivity of an air-core loop antenna can be shown to be proportional to the square root of $A M$, where $A$ is the area of the loop and $M$ is the mass of the metal used in the loop. Put simply, the only way to increase the sensitivity of the antenna, for a given LNA, is to make the antenna either bigger or more massive. But if an antenna is to be extremely light, that implies smaller amounts of thinner wire, which leads to smaller values of $R_{a}$ and therefore $L_{a}$. The opposite is true for extraordinarily large antennas. Empirically, we choose $R_{a}=1 \Omega$ and $L_{a}=0.5-1.0 \mathrm{mH}$, which yields antennas on the scale of meters that are sufficiently sensitive for scientific remote sensing but compact enough that they can be easily deployed in most land locations on the Earth, as well as on ships. ${ }^{129,130}$ But the design principles can be altered with a different starting point for $R_{a}$ if the goal is to make, for example, very small antennas which may be useful for other applications.

Given the conductivity of copper $\left(5.8 \times 10^{7} \mathrm{~S}\right)$, for 16 AWG (American Wire Gauge) wire (1.29 mm diameter), the $1 \Omega$ resistance implies $\sim 76 \mathrm{~m}$ of wire in one loop. For 14 AWG wire (1.63 mm diameter), this leads to $121 \mathrm{~m}$. This length of wire can then be wrapped in a loop any number of times, which drives the inductance of the antenna. For example, the $76 \mathrm{~m}$ length can be a single circular loop with $\sim 24 \mathrm{~m}$ diameter, or two loops with $12 \mathrm{~m}$ diameter. Both have the same resistance but a different inductance. The formulas for the inductance can be found in Refs. 122 and 123. As the number of turns increases (for the same wire length), the inductance also increases. The number of turns can then be increased until a value of $L_{a}$ is obtained that lies between 0.5 and $1.0 \mathrm{mH}$. In this way, we can produce a number of possible antenna designs. We note that the inductance formula makes a couple of assumptions, namely, that the wires are wound in a very tight bundle, but the wires are surrounded by a plastic insulation that doubles the diameter of the wire. This brings the inductance formula roughly in line with a few empirical measurements and lowers the inductance compared to the formula in Refs. 122 and 123, which did not include the added radius of the wire insulation.

A table of selected antenna sizes meeting our $1 \Omega$, $0.5-1.0 \mathrm{mH}$ design spec is shown in Table I, for right isosceles triangle, square, and circular shapes. For the right isosceles triangle, the hypotenuse is assumed to be along the ground, so the height of the triangle is therefore half the base length. For the circular antennas, the base refers to the diameter. All antenna designs shown here have $1 \Omega$ resistance. The loop, when erected, is typically left slightly loose rather than pulling tight so that wind-induced vibrations do not become a factor.

The noise level inherent in the antenna arises from the thermal noise in the wire resistance, which is defined as $\sqrt{4 k_{B} T R_{a}}$, in units of $\mathrm{V} / \sqrt{\mathrm{Hz}}$, where $k_{B}$ is Boltzmann's constant and $T$ is the temperature. This noise voltage is constant with frequency; however, the voltage induced by a magnetic field is
TABLE I. Selected antenna designs.

\begin{tabular}{lcccccc}
\hline \hline Base $(\mathrm{m})$ & AWG & Turns & Area $\left(\mathrm{m}^{2}\right)$ & Mass $(\mathrm{kg})$ & $L_{a}(\mathrm{mH})$ & $S_{0}\left(\mathrm{~T}-\mathrm{Hz}^{1 / 2}\right)$ \\
\hline \multicolumn{7}{c}{ Right isosceles triangle } \\
\hline 201 & 8 & 1 & 10101 & 36.29 & 0.92 & $2.03 \times 10^{-15}$ \\
63.4 & 10 & 2 & 1006 & 14.46 & 1.01 & $1.02 \times 10^{-14}$ \\
26.5 & 12 & 3 & 176 & 5.68 & 0.85 & $3.89 \times 10^{-14}$ \\
10 & 14 & 5 & 25.3 & 2.27 & 0.78 & $1.64 \times 10^{-13}$ \\
2.62 & 16 & 12 & 1.72 & 0.89 & 0.89 & $9.95 \times 10^{-13}$ \\
\hline \multicolumn{7}{c}{ Square } \\
\hline 1.72 & 16 & 11 & 2.98 & 0.89 & 0.92 & $6.24 \times 10^{-12}$ \\
0.57 & 18 & 21 & 0.32 & 0.35 & 0.85 & $3.00 \times 10^{-12}$ \\
\hline \multicolumn{7}{c}{ Circle } \\
\hline 0.30 & 20 & 32 & 0.071 & 0.14 & 0.74 & $9.04 \times 10^{-12}$ \\
0.22 & 20 & 44 & 0.037 & 0.14 & 0.89 & $1.24 \times 10^{-11}$ \\
\hline \hline
\end{tabular}

proportional to frequency. As a result, the signal to noise ratio (SNR) of the antenna coil alone for a given magnetic flux density increases proportionally with frequency. We can calculate the equivalent antenna noise level (or alternatively, the magnetic field for which the SNR is 1) by setting the thermal noise equal to the induced EMF and then solving for $B$. Since the result is proportional to frequency, we can multiply this value by $f$ to obtain a parameter, normalized sensitivity $\left(S_{0}\right)$, in units of $\mathrm{T}-\sqrt{\mathrm{Hz}}$, which defines the quality of the antenna from the perspective of sensitivity. This value is shown in the last column of Table I. We emphasize that the noise level achieved in practice is higher, as the imperfect LNA adds some $\mathrm{dB}$ of noise level to this baseline, so the antenna noise is a lower bound. The noise figure, defined by the LNA, scales the noise of the antenna by a set amount, as a function of frequency. However, the normalized sensitivity is useful as a figure of merit for the antenna since for the LNA we employ, the overall receiver sensitivity as a function of frequency scales linearly with the antenna sensitivity.

The LNA is built with a pair of matched PNP transistors, SSM2220. The design must then balance three somewhat competing goals: (1) a low input resistance so that the flat response with frequency inherent in the antenna is maintained and the total thermal voltage noise (which comes from the series combination of antenna resistance and LNA input resistance), (2) a low input current noise in order to reduce the noise figure, and (3) a wide bandwidth to carry the frequency response up to $500 \mathrm{kHz}$. So we utilize a transformer, whose design principles are described by Refs. 122 and 123. For the LF receiver, the turns ratio of the transformer is 24:56, with the 24-turn side facing the antenna (primary side). The resistance of the SSM220 is biased with $500 \mu \mathrm{A}$ of current, setting the impedance to $\sim 104 \Omega$ on the secondary side, which then yields $19.1 \Omega$ on the primary side. In combination with the $1 \Omega$ antenna resistance, this implies an actual realized system turnover frequency of $3.2 \mathrm{kHz}\left(\omega=\frac{\left(R_{a}+R_{l}\right)}{L_{a}}\right)$, although as shown later, in practice, the receiver is usable down to $\sim 500 \mathrm{~Hz}$. The proper design of the transformer is critical, as other efforts utilizing a transformer for low-impedance amplifiers have not yielded a very good noise figure. ${ }^{131}$ 
On both the primary and secondary sides of the input transformer, the design includes capacitors to suppress the high frequency interference from, for example, AM radio stations well above $500 \mathrm{kHz}$. The cost of this radio-frequency interference (RFI) suppression circuitry, however, is a steeper rise in the noise characteristics at higher frequencies, toward the upper end of the $500 \mathrm{~Hz}-470 \mathrm{kHz}$ passband.

\section{B. Filtering and sampling}

The receiver consists of two stages, one with low-gain trans-impedance amplification to match the impedance out of the transformer and the other providing additional voltage gain designed to set the receiver noise close to the quantization noise floor of the analog-to-digital converter. Both stages are contained within a preamplifier, which is housed in a weatherproof and water-resistant steel box, usually housed outside very close to the antenna itself. After the preamplifier, the signal is then driven along a specialized audio cable (1217B) made by Belden, with four $75-\Omega$ twisted and shielded pairs, and a resistance of $49 \mathrm{~m} \Omega / \mathrm{m}$, rated for up to $2 \mathrm{~A}$ of current. Three of the twisted pairs are used for signal lines, and the fourth one is used for power. The cable is usually passed indoors to a line receiver.

The line receiver has five functions: (1) provide power to the preamplifier, (2) operate the GPS receiver timing module, (3) apply an anti-aliasing filter, (4) digitize the data and send to a recording computer, and (5) reject the common-mode interference that may be picked up on the long transmission line from the preamp. The GPS timing system is described later. The anti-aliasing filter has an upper cutoff of around $470 \mathrm{kHz}$. The analog-to-digital converter, made by National Instruments, is integrated into the line receiver, providing a 16-bit dynamic range ( $96 \mathrm{~dB}$ ) at a $1 \mathrm{MHz}$ sampling rate, and connects to a computer via a universal serial bus (USB) port, along with timestamps from the GPS receiver. The two channels are sampled synchronously, within $\sim 2$ ns of each other (as measured by an oscilloscope). The anti-aliasing filter consists of three cascaded low noise low pass filtering ICs. The circuit can be modified to operate as an 8th order or a 12th order elliptical low pass filter, although in practice we have been using the 12th order almost exclusively. It has a low-pass cutoff frequency of approximately $470 \mathrm{kHz}$. Nominal operating conditions are as a 12th order filter with unity gain to minimize passband ripple and ensure a steep enough roll off to prevent aliasing.

\section{Calibration}

As stated earlier, a given sinusoidal magnetic flux density relates to the induced loop voltage, $V$, via $V=\omega N A B$, where $N$ is the number of turns, $A$ is the area of the antenna, and $B$ is the magnetic flux density amplitude. $N$ and $A$ are precisely known, and we can take into account the impedance in series with the antenna from the LNA as seen on the other side of the transformer at the primary. So at a given frequency, there is a known relationship between the induced voltage and the magnetic flux density. With ferrite-core antennas, there is uncertainty about the magnetic properties of the core, requiring calibration with an actual magnetic field. But for air-core loops, for which this relationship emerges directly from Faraday's law (since the permeability of air is well known), calibration is relatively straightforward with air-core magnetic loop antennas.

Similar to the original AWESOME design, the preamplifier contains a calibration circuit that produces a frequency comb arising from a pseudorandom 1023-bit sequence. The frequency comb components are $\sim 2.5 \mathrm{kHz}$ apart, each of which has a known voltage of $1 \mathrm{mV}$ RMS flowing through a $10 \mathrm{k} \Omega$ resistor into the system, yielding $100 \mathrm{nA}$ injected. Calibration can also be done by manually injecting a single-frequency tone into the receiver and repeating for many frequencies. These signals are done with a "dummy loop" attached, which is a $1 \Omega$, $1 \mathrm{mH}$ inductor-resistor series circuit to mimic the impedance of the antenna without the magnetically induced voltage.

We can associate the $100 \mathrm{nA}$ injected current with a magnetic flux density, as a function of frequency, taking into account the input LNA circuit. By recording the output with an ADC, we can then associate that magnetic flux density with an ADC amplitude. We can verify linearity by scaling the magnitude of the calibration signal and ensuring that the output also scales proportionately. Within the 16-bit resolution of the $\mathrm{ADC}$, we cannot detect any nonlinearity in the receiver's gain. Once the reference calibration signal is related to a magnetic field, we can also characterize the noise floor of the receiver by measuring the RMS levels when the calibration signal is off and the "dummy loop" still connected at the input to terminate the preamplifier input.

\section{RECEIVER CHARACTERISTICS}

The basic receiver frequency response and noise characteristics are shown in Fig. 2. The top left panel shows the gain as a function of frequency, for three different sizes taken from Table I: the $10-\mathrm{m}$ base right isosceles triangle, the $2.6-\mathrm{m}$ base right isosceles triangle, and the $56 \mathrm{~cm}$ length square. These are labeled as large antenna (red), small antenna (blue), and portable antenna (green), respectively.

The antenna does not have impact on the shape of the frequency response of the receiver, so all three show the same characteristics apart from a different scaling factor. The receivers have a low frequency cutoff of below $3 \mathrm{kHz}$, below which the noise levels increase with decreasing frequency. Signal detection is possible well below $3 \mathrm{kHz}$, but with decreasing sensitivity. The system also has a reasonably flat passband between 3 and $400 \mathrm{kHz}$, and then a dropoff until $500 \mathrm{kHz}$ from the anti-aliasing filter.

The top right panel shows the noise characteristics for the same three antennas. The traces show the equivalent magnetic flux density of the noise levels as measured by the analog-todigital converter, using the calibration signal as a reference. This curve includes the noise from the antenna, the preamp, the anti-aliasing card, and all system components. As before, the noise characteristics for all three antennas are identical apart from a scaling factor. The measured noise characteristics include the effect of environmental noise coupling into the exposed electronics in the line receiver box, particularly after the differential amplifier in the preamp and in the line receiver, which is why the curve has some bumpiness and elevated noise bands around 70, 90, 350, and $400 \mathrm{kHz}$. The true receiver noise 


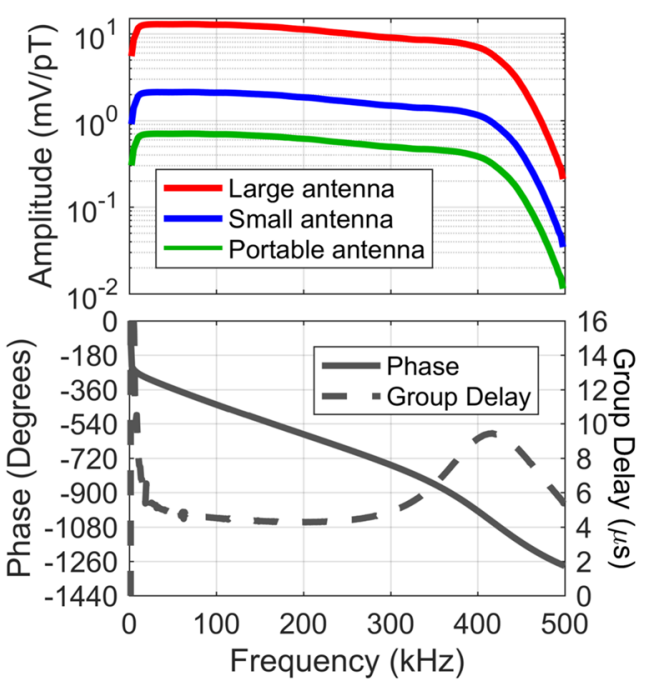

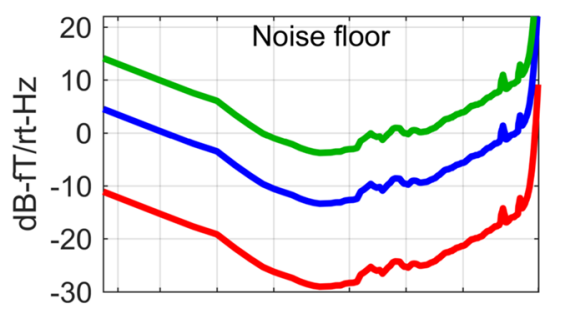

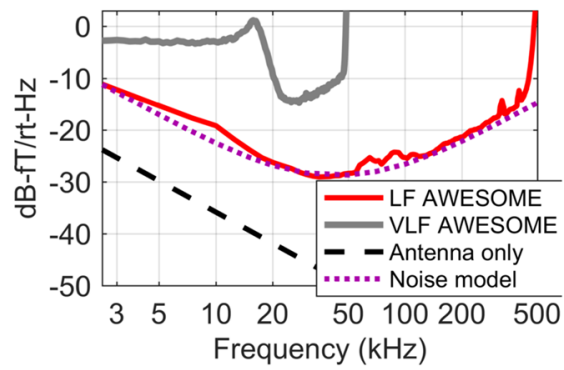

FIG. 2. Amplitude, phase, and noise response of the LF AWESOME, with different antenna sizes and gain settings, as a function of frequency. floor is, more accurately, a smoothed version of the curves shown.

The noise levels for the large antenna are below 0.1 $\mathrm{fT} / \sqrt{\mathrm{Hz}}$ over a frequency band from $\sim 10$ to $230 \mathrm{kHz}$ and reach as low as $0.03 \mathrm{fT} / \sqrt{\mathrm{Hz}}$ around $30 \mathrm{kHz}$. Even for the small antenna, noise levels are below $1 \mathrm{fT} / \sqrt{\mathrm{Hz}}$ for nearly the entire frequency band from 6 to $330 \mathrm{kHz}$. And even the portable antenna noise level is below $1 \mathrm{fT} / \sqrt{\mathrm{Hz}}$ for the VLF/LF transmitter frequency range $(17-120 \mathrm{kHz})$. We can also compare these values to the original VLF AWESOME, as presented in (Ref. 124, Fig. 3). In the bottom right panel of Fig. 2, we reproduce the noise levels from the original VLF AWESOME in gray (for its frequency band below $50 \mathrm{kHz}$ ), for the same large antenna as shown by the red curve. We also show in a dashed line the noise from just the $1 \Omega$ resistance of the antenna, which is how the receiver would behave if the LNA were completely noiseless and ideal. The original VLF AWESOME, for a large antenna, achieved noise levels just barely better than $1 \mathrm{fT} / \sqrt{\mathrm{Hz}}$ below $20 \mathrm{kHz}$. We observe $\sim 10-25 \mathrm{~dB}$ better noise levels for the new LF AWESOME receiver version compared to the original VLF AWESOME, depending on the frequency. Even the small antenna with the LF AWESOME yields better noise performance over nearly the entire frequency band than the large antenna with the previous VLF AWESOME, despite the fact that the small antenna is $\sim 17 \mathrm{~dB}$ less sensitive than the large antenna. The improved sensitivity of the LF AWESOME is likely due to the better circuit board design.

The bottom right panel of Fig. 2 also shows, in a black dashed line, the noise only from the $1 \Omega$ impedance of the antenna, for the large size. The output of a noise model, calculated before the receiver was even built, is shown by the magenta dotted line. The noise model is similar in nature to the one calculated in Ref. 123 but computed for this design. The noise model lines up very well with measurements, save for some portions of the spectrum where environmental noise coupling into the line receiver electronics has a slight measurable effect (at least for the signal levels injected by the automatic calibration tone).

It should be noted that for high-sensitivity receivers, the atmospheric noise floor plays a significant factor in setting the power detection levels. However, unlike at higher frequencies, atmospheric noise in the VLF/LF band is dominated by lightning-generated sferics, ${ }^{132}$ which are highly impulsive, and also a strong function of season and location. ${ }^{133}$ As a result, the noise cannot be characterized as a Gaussian independent process. As a result of the non-Gaussian nature of noise in this band, existing measurements of the RMS atmospheric noise levels, which are well above the sensitivities of the LF AWESOME receiver, do not describe the lowest receiver sensitivity that is valuable. For example, if the noise is modeled as a Poisson arrival process, ${ }^{134}$ then there will be periods in between the randomly arriving impulses where the noise level is quite low, for brief periods of time. Furthermore, there are ways to subtract the impulses from the magnetic field recordings to recover lower atmospheric noise levels. Finally, gradiometric measurements consisting of the difference between two nearby receivers can efficiently subtract most atmospheric noise sources, leaving only receiver noise. As such, there is value in extending the receiver sensitivity well below the RMS atmospheric noise floor.

The gain of the receiver also sets the clipping/saturation level. For instance, the $\sim 12.5 \mathrm{mV} / \mathrm{pT}$ gain of the large antenna in the VLF band implies that a signal with magnetic flux density above $\sim 400 \mathrm{pT}$ causes the receiver to clip since the ADC has a voltage range of $\pm 5 \mathrm{~V}$. This is suitable for quiet sites in remote areas but may not work for sites close to lightning. For comparison, lightning with $30 \mathrm{kA}$ peak current at a $300 \mathrm{~km}$ range can be expected to launch a VLF/LF sferic which arrives at the receiver with $100 \mathrm{~s}-1000$ s of pT of amplitude.

The easiest way to get around this is to use a smaller antenna. For example, the portable antenna has a lower gain by close to a factor of 18.3 (the gain being proportional to the inverse of the normalize sensitivity, or proportional to the product of the area with the number of wire turns). This increases the clip level to $>7300 \mathrm{pT}$, or $7.3 \mathrm{nT}$, which is much more suitable to nearby lightning measurements. Lightning with $100 \mathrm{kA}$ peak current at a $100 \mathrm{~km}$ range can be expected to launch a VLF/LF sferic which arrives at the receiver with 1s-10s of nT. It is worth noting that for a 1-s integration at a noise level of $0.12 \mathrm{fT} / \sqrt{ } \mathrm{Hz}$, the noise amplitude is $0.12 \mathrm{fT}$, which is actually 
$130 \mathrm{~dB}$ below the clip level despite the fact that the receiver is sampled at 16 bits. This reflects the fact that increasingly long integrations allow for increasingly weak signals to be detected, even below the quantization level of the ADC.

Finally, in the bottom left panel of Fig. 2, we show the phase response of the receiver. To calculate this, we take a sinusoidal signal from a function generator and utilize two channels in the ADC. In one channel, we inject the signal directly from the function generator. In the other channel, we inject the signal into the dummy loop which then passes through the entire system. From the phase difference between the two signals as recorded by the computer, we can then infer the phase response, which is shown by the solid gray curve. The dashed gray curve is the group delay, in $\mu$ s. The group delay is defined as the derivative of the phase (in rad) with respect to the angular frequency $(\mathrm{rad} / \mathrm{s})$, which has units of time. The receiver is fairly linear between 1 and $350 \mathrm{kHz}$, with a group delay of 4-5 $\mu$ s. As the frequency nears the cutoff of the anti-aliasing filter, the group delay rises to about $10 \mu \mathrm{s}$. The group delay, or the propagation delay of signals through the receiver, is dominated by the contribution of the Anti-aliasing Filter (AAF). The AAF consists of 12 orders of resistor-capacitor combinations so impede (i.e., slow) the transfer of voltage/current from one side of the AAF to the other.

\section{A. Timing accuracy}

A highly precise sampling clock is desirable for two reasons: (1) accurate measurement of the phase of VLF transmitting beacons and (2) interferometric or other multi-site measurements which require a consistent time reference.

There are low-cost commercial GPS receivers that provide a steady 1 Pulse Per Second (PPS) reference accurate to $\sim 100$ ns. Any given 1 PPS GPS pulse cannot be relied onto be accurate. Although most pulses occur within $100 \mathrm{~ns}$ of the start of the second, some spurious pulses may be much less accurate. So while a single pulse cannot on its own condition a clock, a long train of many can be. Our goal is to use a train of 1 PPS GPS pulses to produce an accurate $1 \mathrm{MHz}$ sampling clock.

A number of more expensive timing devices are available on the market, but they are optimized for a highly accurate frequency reference rather than a timing reference. But there is a fundamental trade-off between the accurate frequency and accurate timing. As no frequency reference is perfect, and since the phase error is the integral of the frequency error as a function of time, even a small error in frequency will eventually lead to a significant and measurable phase error. The fastest way to then correct that timing error when it gets too big is to change the frequency, but this violates the goal of a frequency reference, so the correction is done slowly. As such, frequency references may sacrifice some timing accuracy to avoid larger adjustments in frequency. Since our goal is to optimize for timing accuracy, not frequency accuracy, we have engineered our own solution (and as a bonus it is much less expensive than commercially available products).

We have therefore custom-built a timing adjustment algorithm that conditions a voltage-controlled oscillator (VCO) to be highly accurate. The algorithm is spread across two devices, a microcontroller and a Complex Programmable Logic Device

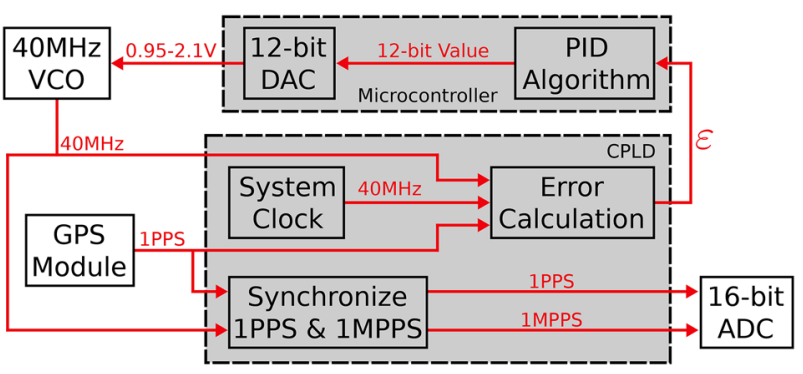

FIG. 3. (Top) Block diagram of the timing error correction circuit.

(CPLD). A diagram of the entire timing adjustment system is shown in Fig. 3. The CPLD has two tasks: output the stable and synchronized 1 MPPS sampling clock and 1 PPS sampling trigger and measure the timing error of the VCO. Three signals (clocks) are used by the CPLD to complete these tasks: a 40 $\mathrm{MHz}$ VCO signal that is GPS-conditioned, a second independent $40 \mathrm{MHz}$ system clock that is NOT GPS-conditioned, and a 1 PPS marker from the GPS receiver.

Generating the two-phase locked output signals from the CPLD, the 1 MPPS sampling clock, and the 1 PPS sampling trigger is accomplished by dividing the $40 \mathrm{MHz}$ VCO by 40 and $40 \times 10^{6}$, respectively. Division of these signals is closely (typically within $\pm 40 \mathrm{~ns}$ ) aligned with the input GPS 1 PPS signal. However, the input 1 PPS and the output 1 PPS are not perfectly phase locked because some phase jitter (typically within \pm 40 ) exists in the GPS 1 PPS signal.

In practice, due to the inherent uncertainty of each 1 PPS pulse from the GPS receiver, there may not be exactly $40 \times 10^{6}$ ticks of the $40 \mathrm{MHz}$ VCO between each 1 PPS, even if the $40 \mathrm{MHz}$ VCO is perfect. Properly adjusting for this error, without overadjusting, is thus critical. The second process in the CPLD measures the timing error $\varepsilon$ every second to enable clock frequency corrections. In this case, $\varepsilon$ is defined as the delay between the rise of the 1 PPS signal from the GPS receiver, and the rise of the 1 PPS sampling trigger (which is equivalent to the 40 millionth cycle of the VCO). The bottom panel of Fig. 3 displays a block diagram of the method used to quantify $\varepsilon$. The $40 \mathrm{MHz}$ VCO clock is first divided into a $100 \mathrm{kHz}$ signal. A lower frequency makes the rising edge comparison simpler as there is a greater period between rising edges at $100 \mathrm{kHz}$ and allows for more dynamic range in the measurement of $\varepsilon$. The phase detector is triggered when the rising edge of the GPS 1 PPS occurs, enabling the counter. The counter then begins to count the number of $40 \mathrm{MHz}$ system clock cycles, continuing until the phase detector receives a rising edge from the $100 \mathrm{kHz}$ signal. At this point, the counter stops and holds its value for the microcontroller to read. This value, however, is not the measured error $\varepsilon$ because the counter measures the time difference between rising edges of the GPS 1 PPS and the next $100 \mathrm{kHz}$ rising edge. Instead, $\varepsilon$ is the value held in the counter less than 200 (half of a period at $100 \mathrm{kHz}$ ). This means the algorithm attempts to phase lock the GPS 1 PPS and the falling edge of the $100 \mathrm{kHz}$ signal (which occurs at the same time as the rising edge of the output 1 PPS sampling trigger), and the ideal counter value is 200 . Any deviation from 200 is $\varepsilon$, rounded to $25 \mathrm{~ns}$ resolution (due to the $40 \mathrm{MHz}$ system clock). 
The microcontroller, in our case an Atmel XMEGA$128 \mathrm{~A} 4 \mathrm{U}$, determines the required clock adjustment each second. The choice of a control algorithm depends on the specific system performance and how it responds to feedback. In our case, important components are our VCO and GPS 1 PPS and how they respond together to thermal drift, control feedback, etc. We empirically determined that the best control performance is achieved with a proportional-integralderivative (PID) control algorithm. This is in contrast to systems which may achieve better performance by using only one or two terms of the PID algorithm. We use empirically determined coefficients to find the three PID terms, the absolute error, the running integral, and the derivative. The coefficients are optimized with the goal of achieving a balance between two competing goals: (1) mitigating the effect of spurious 1 PPS pulse times, and (2) responding quickly should the VCO change frequencies due to, for example, temperature changes. The three PID terms are then summed together to produce a 12bit value that is fed into a digital-to-analog converter (DAC), which is internal to the microcontroller. Changes in the 12-bit value fed into the DAC will either increase or decrease the frequency of the $40 \mathrm{MHz} \mathrm{VCO}$, allowing the PID algorithm to accurately phase lock the $40 \mathrm{MHz}$ VCO with the GPS 1 PPS.

There are two ways to measure and verify the error in our sampling clock, both of which are shown in Fig. 4. The first technique uses an existing VLF transmitter, in this example, the NLK transmitter at $24.8 \mathrm{kHz}$, broadcast from Jim Creek, WA, and detected at Baxley, GA. The left panel shows the phase of the transmitter over a $24 \mathrm{~h}$ period on $\pi$ day, 2017 (the algorithm for calculating the phase is described later). The daytime period from 12 to 24 UT is very stable, as the Sun's ionization is predictable and constant. At 00 , UT is a transition to nighttime, which typically demonstrates a significant phase shift as the ionospheric electron density reduces, after which the propagation conditions are much less stable. We can utilize the stable daytime portion (12-24 UT) to test out the phase stability of the receiver.

The VLF transmitters do not have a precise phase reference; hence, the phase at the source wanders fairly slowly, on minutes-long time scales. However, on time scales of seconds, the phase is relatively constant. On the other hand, corrections to the $\mathrm{VCO}$ are done each second. In the previous AWESOME receiver, the VCO was intentionally left slightly slow so that there would never be an extra sample in a second. This resulted in the measured phase drifting forward during each second, which was then reset with each second marker. This timing drift was on the order of $10 \mathrm{~s}$ of ns and could be seen via superposed epoch analysis, or stack-averaging repeating time windows of the phase data, which accentuates any effect that is periodic at a multiple of the segment length. This can be seen in Fig. 4 of Ref. 124.

The new timing adjustment algorithm on the LF AWESOME eliminates this glitch and does not by design keep the clock inaccurate. The center panel of Fig. 4 shows the same superposed-epoch analysis of the 12-h daytime period shown, and there is no evidence of a drifting clock on the time scale of seconds or shorter, with relative timing accuracy at least down to a few ns.

The third panel of Fig. 4 shows a separate test of the clock's accuracy. A much more expensive GPS-conditioned Microsemi clock is purchased, which is rated to have an absolute accuracy of $25 \mathrm{~ns}$. We set up two GPS antennas, side by side, and measured (with an oscilloscope) the delay time between our $1 \mathrm{MHz}$ sampling clock, and the one provided by the Microsemi unit. The distribution of errors is shown on the right. The RMS error is $15 \mathrm{~ns}$, which is in line with the timing specification for the Microsemi unit so is probably dominated by the error in that unit. So we can say that the error in our custom clock circuit is at least as good as that of the Microsemi and probably better.

\section{B. Sample data}

Figure 5 shows some sample data from the receiver, taken at the Pisgah Astronomical Research Institute, or PARI, near Rosman, NC. The top left panel shows data in the form of a spectrogram. The data are divided into small segments of time, in this case $1 \mathrm{~ms}$, and a Fourier transform is performed on each window. Each time segment forms one column in the spectrogram, with the color indicating the strength of the magnetic field, calibrated at that frequency within that window.

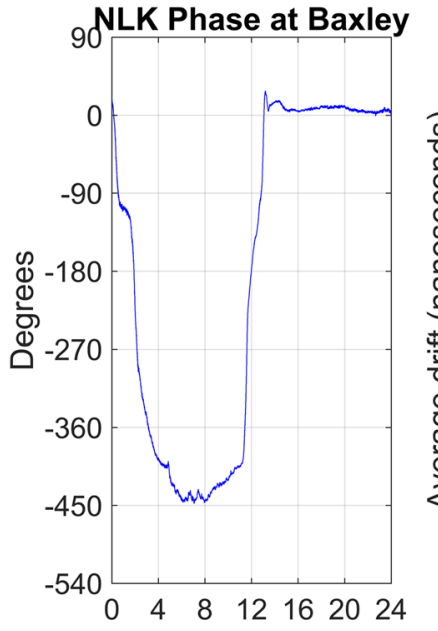

Time (UT) on 14-Mar-2017
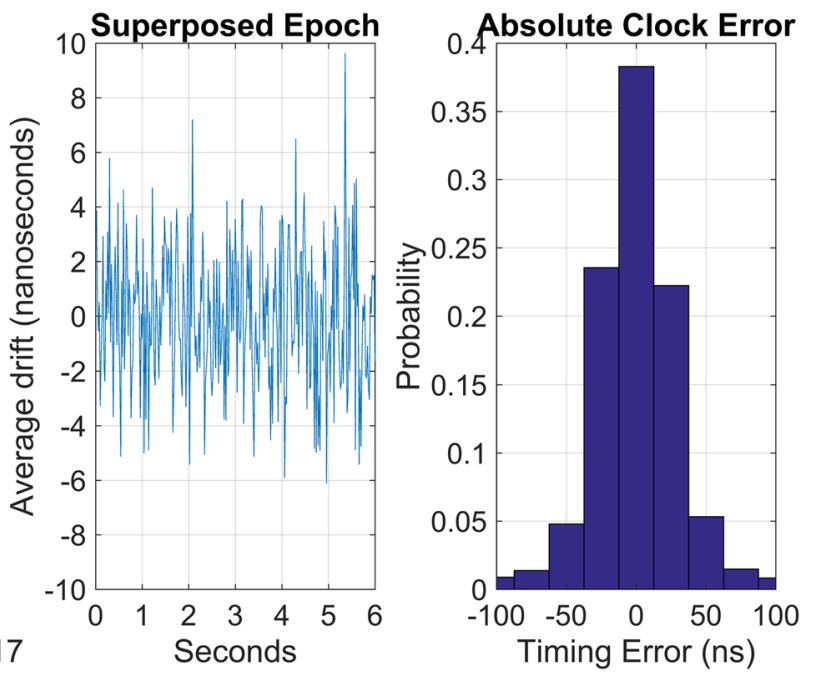

FIG. 4. VLF transmitter phase over a 24-h period, and the timing error of the sampling clock measured in two ways. 


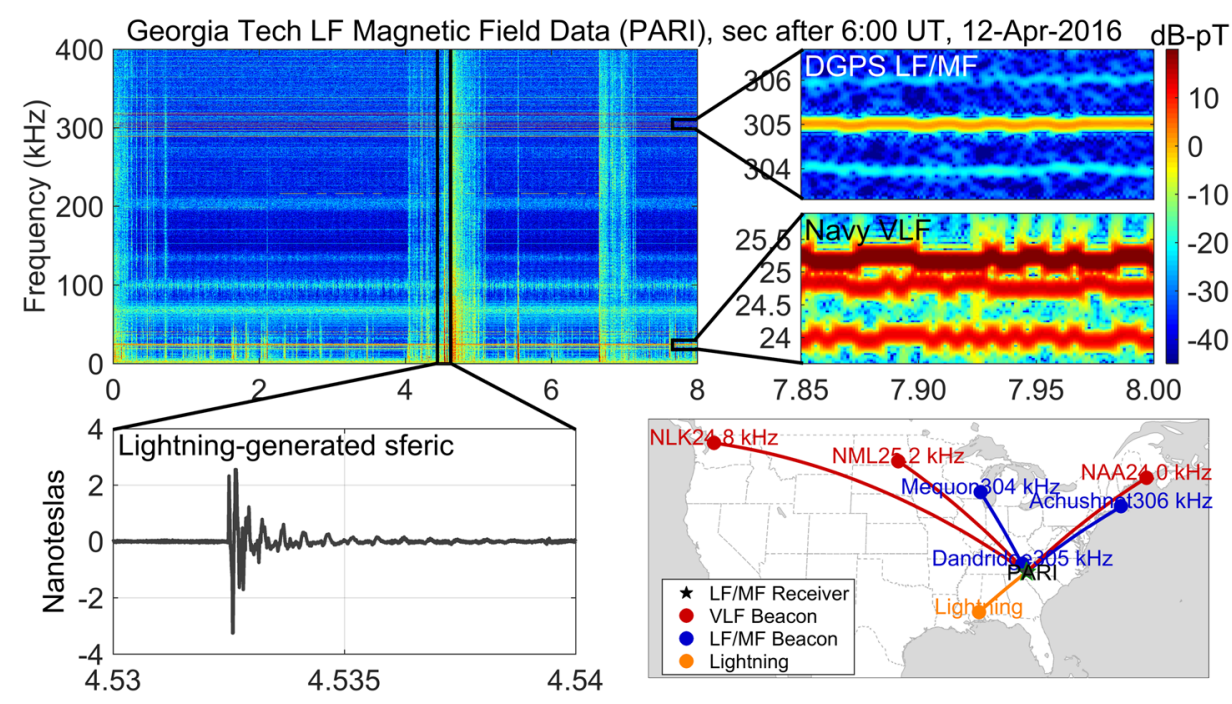

FIG. 5. An example of LF/MF data.

The horizontal axis shows $8 \mathrm{~s}$ of data on April 12, 2016 at 0600 UT, with the vertical axis showing frequency from DC up to $400 \mathrm{kHz}$. Since the window size is $1 \mathrm{~ms}$, the frequency resolution is $1 \mathrm{kHz}$.

The vertical lines in the spectrogram are radio atmospherics from lightning, or "sferics." These may originate from distant lightning many thousands of km away. One such sferic is magnified in the lower left panel, which shows a brief time-domain magnetic field variation over a $10 \mathrm{~ms}$ period. While most sferics last less than $1 \mathrm{~ms}$, this one is an example of a "tweek," or a sferic that rings at close to the waveguide cutoff frequency $(\sim 1.8 \mathrm{kHz})$. This particular tweek originated from a thunderstorm in southern Mississippi, as shown in the map in the lower right panel. The PARI site is very electromagnetically quiet, being not near a city. Although we have developed techniques to remove $60 \mathrm{~Hz}$ power line harmonic radiation, ${ }^{135}$ those are not needed here. It is essentially impossible to find an electromagnetically quiet location on or near the surface where these lightning-generated sferics are not scattered prominently throughout the magnetic field recording.

The horizontal lines in the spectrogram are VLF and LF radio beacons. There are roughly a dozen or so VLF transmitters between 18 and $26 \mathrm{kHz}$, most of which are used by naval forces for submarine communications. On the right is a thumbnail showing three such transmitter signals, the NAA transmitter $(24.0 \mathrm{kHz})$ from Maine, the NLK transmitter $(24.8 \mathrm{kHz})$ from Washington State, and the NML transmitter $(25.5 \mathrm{kHz})$ from North Dakota. These transmitter locations are shown in the map in the lower right panel of Fig. 5, along with the receiver location. For each transmitter, the spectrogram shows the frequency modulating up and down, reflecting transmission of $1 \mathrm{~s}$ and $0 \mathrm{~s}$. There are also a set of beacons between 285 and $325 \mathrm{kHz}$, a network known as the Nationwide Differential GPS system, which broadcasts ionospheric GPS errors to allow GPS location corrections to be made in real time even as space weather and ionospheric disturbances affect the GPS signal. The upper right thumbnail shows three such beacon signals, at 304,305 , and $306 \mathrm{kHz}$, originating from Mequon, Wisconsin, Dandridge, Tennessee, and Achushnet, Massachusetts, respectively.

\section{TRANSMITTER DEMODULATION}

Figure 5 shows examples of VLF and LF/MF transmitter signals that are modulated with minimum-shift keying (MSK), typically at 100 or 200 baud.

This technique may be viewed either in terms of a continuous-phase frequency-shift keying (FSK) signal or as a variant of quadrature phase-shift keying (QPSK). From the FSK perspective, this technique involves shifting the frequency either above or below a center frequency while keeping the phase continuous to represent " 1 " or " 0. " The bit period corresponds to the time it takes for the phase to shift by $90^{\circ}$. Viewed as a variant of QPSK, in-phase (I) and quadrature (Q) binary streams each encodes at half the overall baud rate and uses a half sinusoid modulation and staggered bit transition boundaries such that the overall envelope remains constant. We now detail how this signal is demodulated in the context of multi-mode propagation below the ionosphere, with the goal of enabling remote sensing applications.

The mathematics of MSK demodulation is well established. This section uses notation developed in (Ref. 136, Appendix A), which provides a thorough mathematical treatment of single-mode MSK demodulation. Existing MSK discussions focus on single-mode problems, ${ }^{137-139}$ but in the context of VLF/LF ionospheric remote sensing, there are two additional problems: the multipath and the non-Gaussian noise environment.

\section{A. Multimode propagation}

For carrier frequencies above the first cutoff frequency in the Earth-ionosphere waveguide, multiple modes may exist with different amplitudes and phase and group velocities. Separating these multiple modes can in principle be done with arrays of receivers, ${ }^{140,141}$ which established experimentally that multi-modes exist and can be separated. But it is worth exploring what information can be extracted with one receiver measurement.

If the group velocities of each constituent mode are equal so that the bit transition times are synchronized, the total received signal is indistinguishable from a single mode whose 


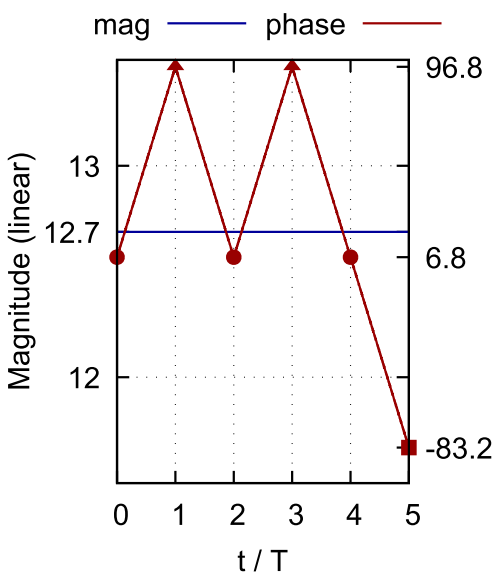

96.8

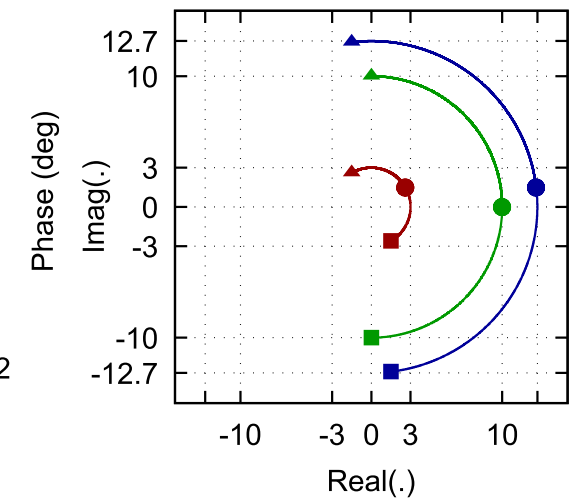

FIG. 6. Five-bit segments of an MSK signal consisting of two modes with the same bit transition time. The amplitude and phases of components $\mathrm{a}$ and $\mathrm{b}$ are $A_{\mathrm{a}}=10$, $\phi_{0 \mathrm{a}}=0, A_{\mathrm{b}}=3$, and $\phi_{0 \mathrm{a}}=30^{\circ}$. The three different phase offsets at the bit transition times on the phase signal (left panel) are marked with a different shape. The same shapes mark the corresponding locations on the phasor plot of the constituent and total signal (right panel). amplitude and phase are determined by the vector sum of the phasors of the constituent modes. This effect is illustrated in Fig. 6, which shows amplitude, phase, and phasor plots of five bits for a simulated signal with two modes a and b, where the amplitudes and phases are given in the figure caption. Since the bit transition times are aligned, the total phasor is given by

$$
z_{\mathrm{T}}(t)=\left(A_{\mathrm{a}} e^{j \phi_{0 \mathrm{a}}}+A_{\mathrm{b}} e^{j \phi_{0 \mathrm{~b}}}\right) \exp \left[j \phi_{\mathrm{MSK}}(t-\tau)\right] .
$$

Hence the total amplitude and carrier offset are

$$
\begin{aligned}
& A_{\mathrm{T}}=\left|z_{\mathrm{T}}(t)\right| \simeq 12.687, \\
& \phi_{0 \mathrm{~T}}=\angle\left\{z_{\mathrm{T}}(t) \exp \left[-j \phi_{\mathrm{MSK}}(t-\tau)\right]\right\} \simeq 6.79^{\circ} .
\end{aligned}
$$

The amplitude and phases of the two modes, and the sum, are clearly seen in the right panel of Fig. 6 .

If the two modes also have different group velocities, then in general the bit transition times are not synchronized. In this scenario, the phasor from the two modes changes direction at slightly different times at the bit transition. The net effect is a rounded "corner" transition when the phase derivative changes sign, and an amplitude that is also dependent on the sign of the phase derivative. A simulated example is shown in Fig. 7, which uses the same amplitude and carrier offsets shown in Fig. 6, but in this case mode $b$ has an additional group delay of $0.1 T$ compared to mode $a$.

This relative bit transition time difference is likely an order of magnitude larger than real MSK signals propagating in the Earth-ionosphere waveguide. If two modes propagate with a group velocity difference of $\delta v$, then to first order the bit transition time is given by $d / v(\delta v / v)$, where $d$ is the total distance and $v$ is the average velocity. Assuming a total propagation time of $d / v=10 \mathrm{~ms}$ and $\delta v=0.01 c$, the time difference would be at most $100 \mu \mathrm{s}$, which is just $0.02 \mathrm{~T}$ at 200 baud.

The left panel of Fig. 8 quantifies the phase estimation in a multi-mode environment. We have generated 10 -s of synthetic MSK data but made two modes with the same bit stream and summed them together and then applied the MSK phase estimation algorithm. We repeated this many times, varying the ratio between the dominant mode (Mode 1) and the secondary mode (Mode 2). The carrier phase of Mode 1 is taken to be $30^{\circ}$ in this example, and for Mode 2 , it is $60^{\circ}$. There is no noise applied in this part. The horizontal axis shows the ratio between Mode 1 and Mode 2, and the vertical axis is the group delay between the two modes.

The estimated carrier phase is independent of the group delay between modes. However, the estimated carrier phase of the sum of the modes is a function of the amplitude ratio between the two. For instance, when the modes are equal, the estimated phase is $45^{\circ}$, which is halfway between the carrier phases of the two modes. When the ratio of the two modes is $6 \mathrm{~dB}$, the estimated carrier phase is $40^{\circ}$, which is $\frac{2}{3}$ of the way between the two. In short, it appears that the MSK phase estimation is an average of the phases of the many underlying modes, weighted by their relative amplitudes.
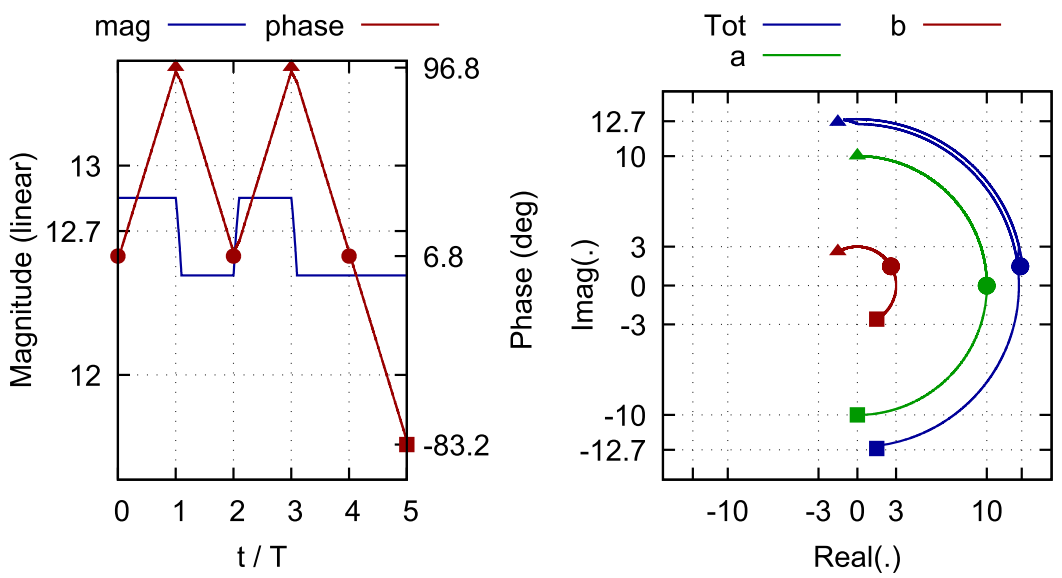

FIG. 7. Amplitude, phase, and phasor plots using the parameters from Fig. 6 , where $\mathrm{b}$ has an additional 0.1 Tbit transition delay. 


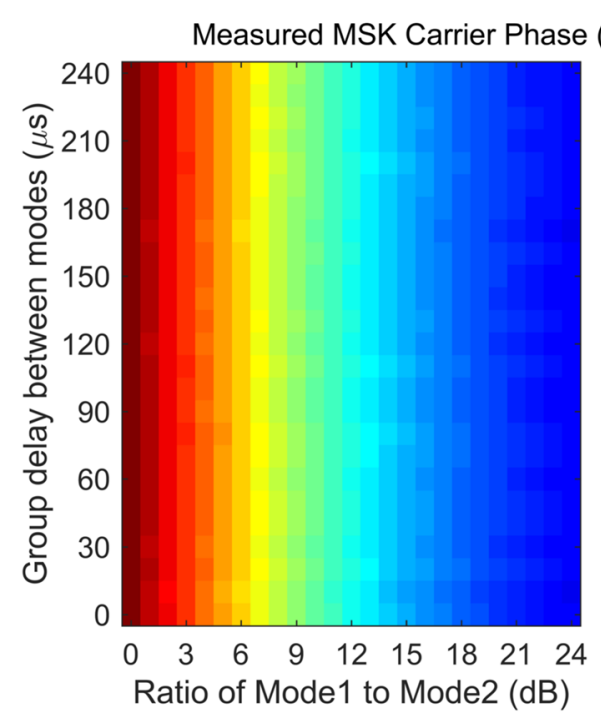

\section{B. Additive Gaussian noise}

Figure 9 shows the same signal of Fig. 6, with an additive white Gaussian Noise source with mean 0 and standard deviation of 0.127. The finite Signal-to-Noise Ratio (SNR) introduces uncertainty to the amplitude, carrier phase, and bit transition time estimates. In this case, the SNR is $40 \mathrm{~dB}$.

Figure 10 shows the carrier phase and amplitude root mean squared error (RMSE) for a signal with $A=12.687$ and $\phi_{0}=6.79^{\circ}$ with varying SNR levels using three different integration windows. The bit sequence is set to be random with a uniform probability for each bit. The carrier frequency is set to $21.4 \mathrm{kHz}$, and the total signal duration is $10 \mathrm{~min}$. For each integration duration, the RMSE for both the phase and amplitude increases as the SNR decreases. For a given SNR, a longer integration window reduces the measurement error. In principle, there is no limit to the length of the integration time since the timing algorithm keeps the receiver locked with GPS within 15-20 ns indefinitely.

The right half of Fig. 8 shows the algorithm's performance when noise is added. We again generate synthetic multi-mode MSK signals and then run it through the demodulation algorithm. The horizontal axis is the ratio of the two modes. The vertical axis is the SNR, defined as the ratio of signal-to-noise within a $\pm 200 \mathrm{~Hz}$ bandwidth around the carrier. The noise is Gaussian so essentially has zero phase at all frequencies.

At high signal to noise ratios, the estimation follows that of the left half of Fig. 8. Starting when the SNR is below $\sim 3 \mathrm{~dB}$, the estimation changes, as the phase of the noise (i.e., $0^{\circ}$ ) starts to increasingly affect the measurement. As such, we can establish an SNR limit of $3 \mathrm{~dB}$ (in the $400 \mathrm{~Hz}$ band), and any mode above that level may contribute meaningfully to the phase estimation, which is quantified by the right panel of Fig. 8.

\section{Additive impulsive noise}

We now discuss the effect to additive impulsive noise. VLF/LF data have noise levels typically dominated (if not by cultural noise) by sferic energy from lightning strokes, and to quantify the MSK algorithm performance against both Gaussian noise and impulsive noise, we do a direct test with simulated data.

Figure 11 shows on the left a series of MSK phase estimates with 1-s of simulated data. We have manually added impulsive energy to the dataset, randomly distributed in power and time. Along the horizontal axis, we have varied the ratio of the total impulsive energy in the $400 \mathrm{~Hz}$ bin to the energy of the main MSK signal (200-baud, one mode, with carrier
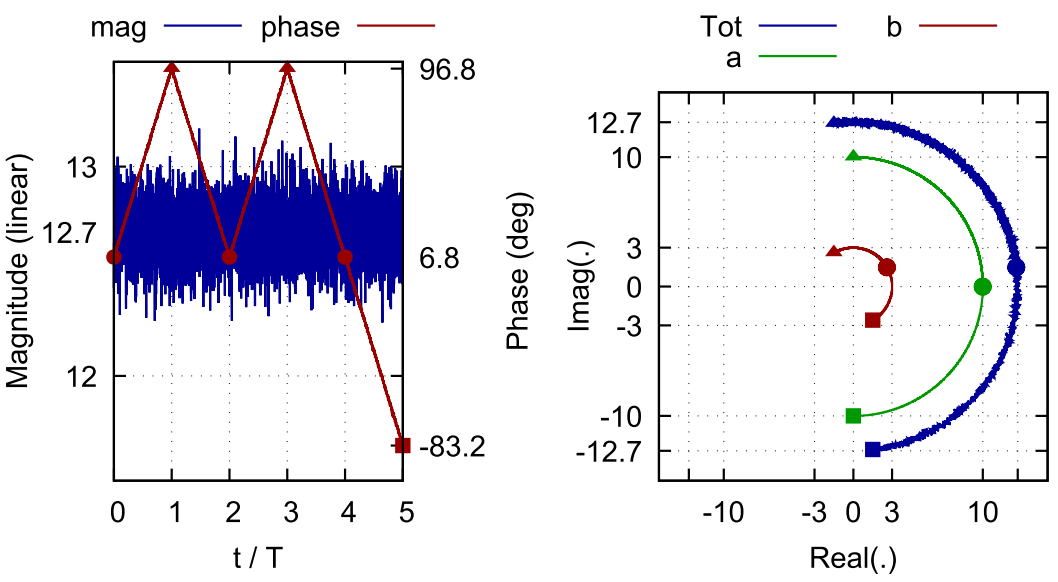

FIG. 9. Amplitude, phase, and phasor plots using the parameters from Fig. 6, where the total signal has a finite $(40 \mathrm{~dB}) \mathrm{SNR}$. 

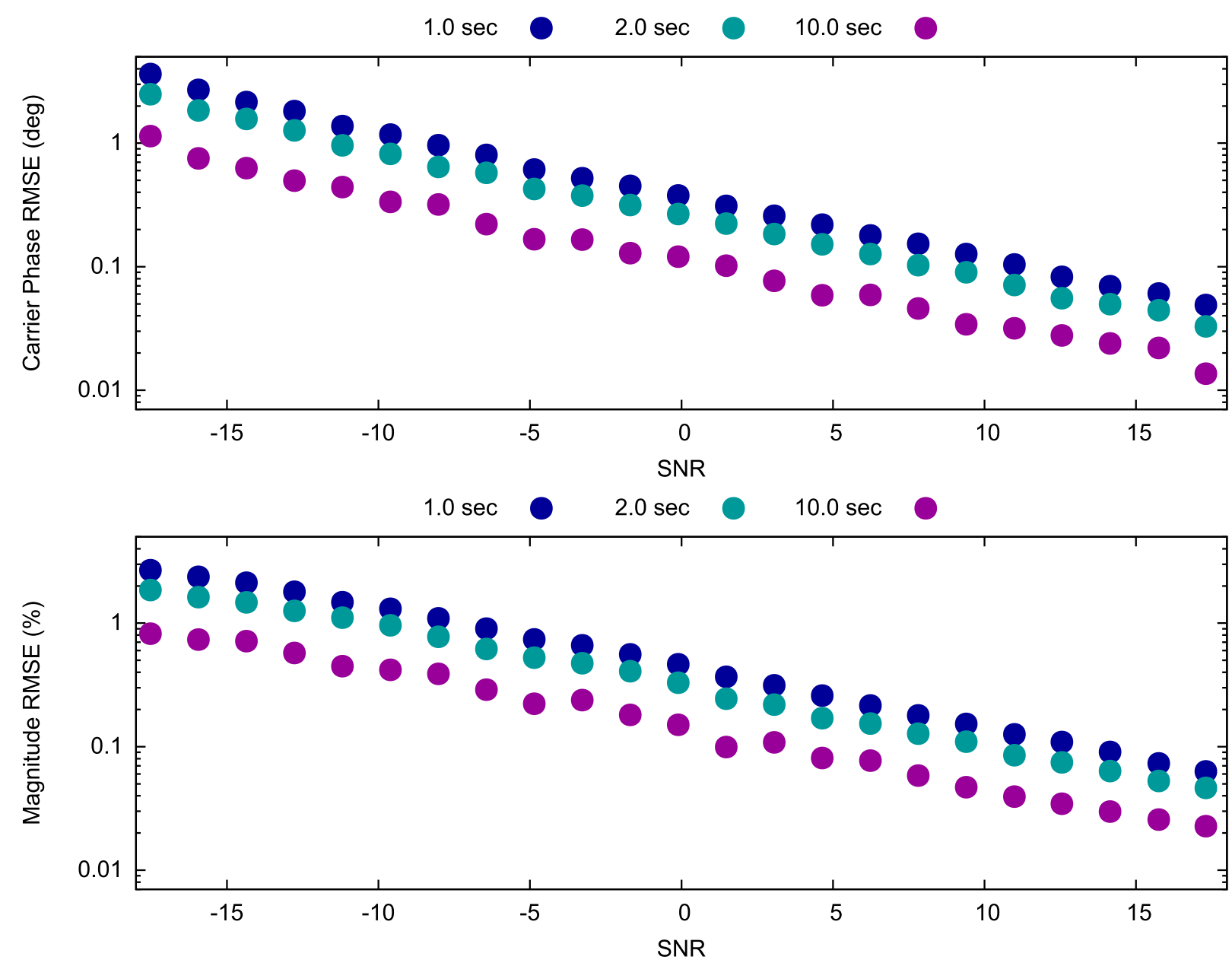

FIG. 10. Root mean squared error (RMSE) of the carrier phase (top panel) and amplitude percent error (bottom panel) estimates versus SNR, in $\mathrm{dB}$, for three different integration window parameters.

phase at $0^{\circ}$ ). The vertical axis is the SNR of the MSK signal compared to the Gaussian noise.

The impulsive noise essentially makes for a random number to be added to the phase measurement. As expected and shown earlier, the performance as the SNR worsens can be seen to hold pretty well until the SNR dips below $0 \mathrm{~dB}$.
The algorithm, however, seems to perform fairly well as impulsive noise increases. Even when the energy in the impulsive noise is significantly stronger, the MSK phase estimates remain fairly reliable. The right plot shows the same except $10-\mathrm{s}$ of data are taken, and an average of the $10 \mathrm{~s}$ is plotted.

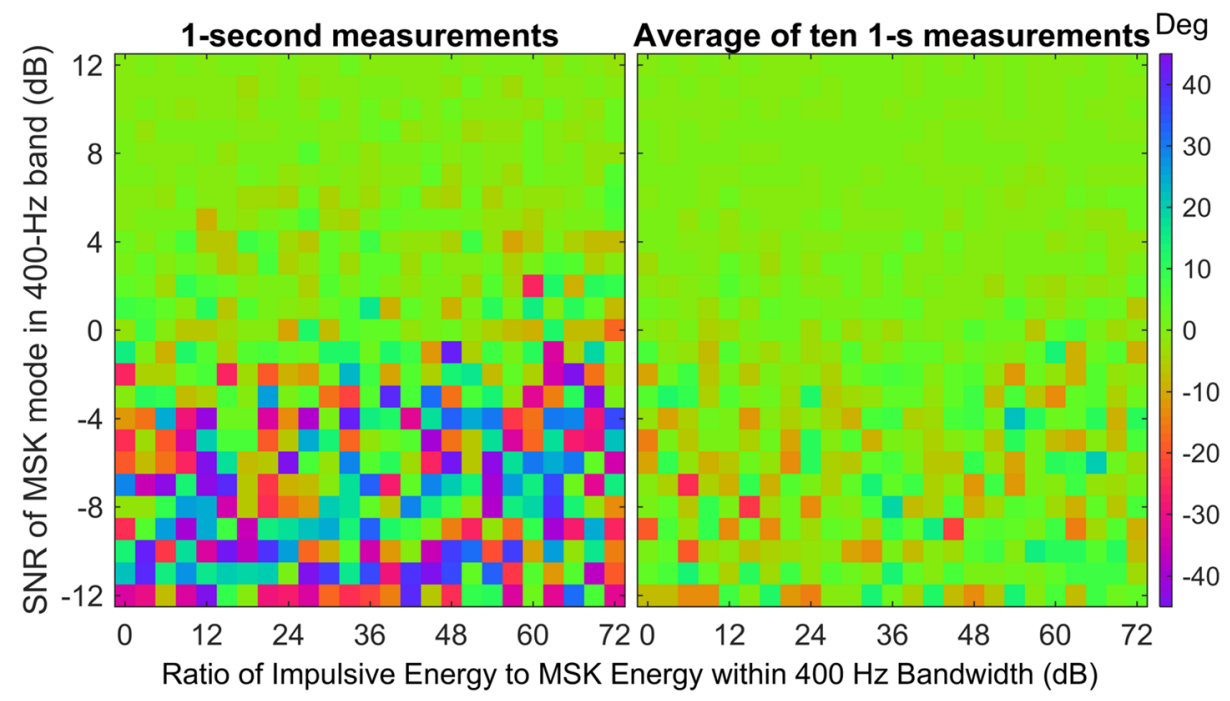

FIG. 11. Phase estimation for the impulsive noise case. Left: Single-run phase estimates as a function of SNR to the Gaussian noise (vertical axis) and SNR between impulsive noise to the MSK energy (within a 400 $\mathrm{Hz}$ bandwidth). Right: The same except using an average of 10 1-s measurements. 
NAA (24.0 kHz) at Baxley, 13-Jan-2017
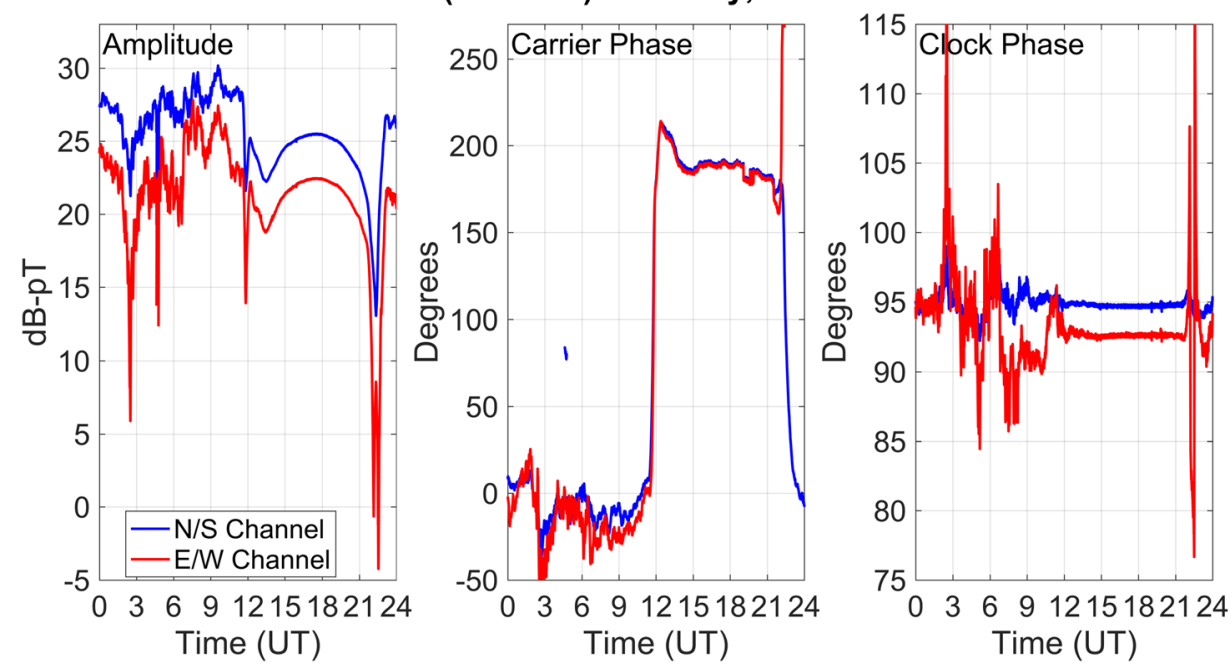

FIG. 12. Transmitter parameters in linear form after MSK demodulation.

\section{Example data}

Figure 12 shows our application of the MSK demodulation algorithm to VLF transmitters. The data shown are $24 \mathrm{~h}$ of data from 13 January 2017, of the NAA transmitter detected at a receiver at Baxley, in southern Georgia. The left panel shows the amplitude of each of the two orthogonal antennas, over the $24 \mathrm{~h}$ period. The convention here is that the North/South (N/S) antenna is sensitive to transverse magnetic (TM) waves arriving from either the north direction or the south direction, which actually means that it picks up the magnetic field component along the east/west axis. The East/West (E/W) antenna is sensitive to TM signals from the east/west direction and picks up the magnetic field component along the north/south axis.

The diurnal signal shows a classic and well-known variation, in which the signal is steady and slowly varying during the daytime, resulting from the sun rising, reaching a peak, and descending along the propagation path. The signal is much more chaotic during the nighttime, and between the two is a very unsteady period during the day/night terminator transition time. Since this path is mostly north/south, the transition from day to night is relatively short. The middle panel shows the carrier phase of the same two antennas, showing a phase advance during the transition to nighttime. The right panel shows the clock phase, which unlike the carrier phase shows roughly the same phase on average between daytime and nighttime, but a much less steady signal during nighttime. We emphasize that clock phase variations are not due to the internal clock within the receiver, which as shown earlier is very stable. Rather, it is due to the "apparent" group delay of the sum of many modes.

The group delay has been investigated before as an ionospheric diagnostic, ${ }^{142,143}$ essentially by looking at the difference in phase between the two frequencies that under MSK transmissions since each may respond differently to an ionospheric disturbance, particularly in a multimode environment.

During the daytime, the signal is often dominated by one mode, whose group delay is constant, and this group delay defines the difference in phase between the upper and lower MSK frequencies. In the single-mode case, the "apparent" group delay is the same as the physical group delay of the actual propagating wave. During nighttime, when multimode mixing is much more common, the coherent addition of many modes can alter the phase difference between the upper and lower frequencies so that the "apparent" group delay cannot be directly linked to a physical delay.

\section{APPLICATIONS}

We now describe a few selected applications of the LF AWESOME within lightning and ionospheric remote sensing.

\section{A. Lightning detection at a global range}

Figure 13 shows a remarkable example of VLF sferics emitted by lightning echoing around the world multiple times. The top left panel shows a global map with the measurement setup. Data are collected from an LF radio receiver site outside Juneau, Alaska. The site is extremely quiet electromagnetically, being many miles from the nearest power line. On 10 September 2017, at 23:33:42.333735 UT, a lightning stroke occurred in the south Indian Ocean that was detected by the GLD360 network. The stroke had an incredible peak current of $+1021 \mathrm{kA}$, as estimated by the network. It is worth noting that such intense lightning currents are rare in general but are surprisingly more common over the ocean, even though lightning itself is much more common over land. ${ }^{11,130}$

The red panel below the map shows the signal detected at Juneau along the most direct path, $\sim 18 \mathrm{Mm}$ away. The sferic has $\sim 400$ pT intensity. The blue curve shows the sferic detected along the antipodal path the other way around the world, which is $\sim 22 \mathrm{Mm}$ away. The delay time is slightly longer, but the top right panel shows that a sferic is indeed detected at the receiver. The purple and green panels show the direct and antipodal paths, respectively, after another round-the-world propagation trip, but all are detected fairly easily by the receiver due to the high sensitivity of the amplifier and the electromagnetically quiet site. It is worth noting that the distance from the source to the receiver is fairly close to halfway around the world so that there is actually some focusing occurring as various antipodal paths converge. As such, in this region, there 

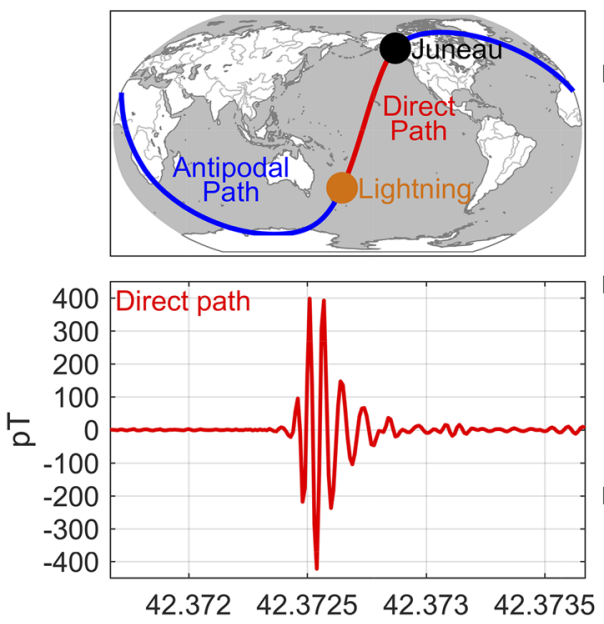

Seconds after 2017-Sep-10 23:33 UT
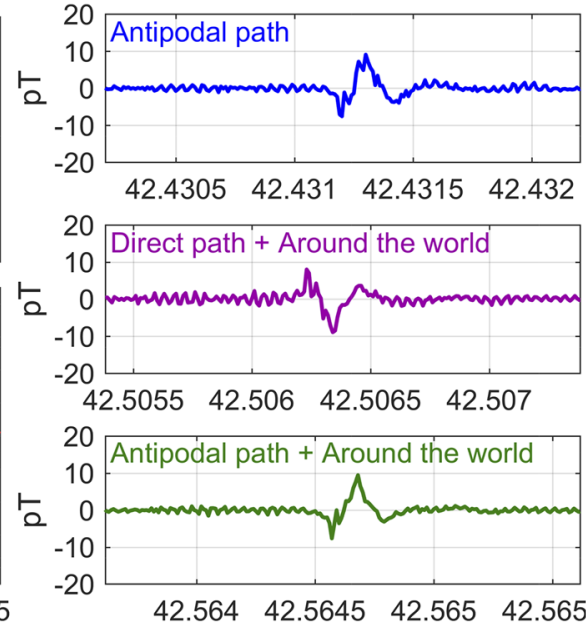

FIG. 13. A lightning stroke echoing around the world. is actually some amplification that makes the antipodal signal stronger and affects the apparent attenuation with distance in complicated ways. In addition, propagation over the Antarctic ice sheets has an impact on the sferic propagation for all but the direct path. We have applied some signal processing to remove interference from power lines ${ }^{135}$ and from VLF transmitters (Ref. 144, Chap. 3). But even with other noise sources (such as LF/MF transmitters to be discussed later), the detection threshold is $\sim 0.5 \mathrm{pT}$, despite the small $1 \mu$ s sampling time.

It is worth noting that the round-the-world echo from lightning strokes has been observed for decades by workers looking at the Schumann resonance band, ${ }^{145,146}$ in which the global Earth-ionosphere cavity has a resonance frequency of $7-8 \mathrm{~Hz}$ and its multiples, which has very interesting radio science applications. ${ }^{147}$ This thunderstorm-associated event is known as a Q-burst. ${ }^{148,149}$

\section{B. lonospheric remote sensing with LF sferics}

In addition to VLF narrowband beacons, sferics from lightning are a useful ionospheric diagnostic, as described in the Introduction. Since the ionosphere is highly sensitive to solar flares, it follows that changing solar flux should in turn be observable in received sferics. This is clearly observed in Fig. 14 by the changing time and spectral characteristics of received sferics at PARI station $(35.20 \mathrm{~N}, 82.87 \mathrm{~W})$ in North Carolina, US. The example representative sferics are processed according the technique discussed in Ref. 150. The example storm systems took place over Panama throughout the afternoon which allowed for long-term probing of the ionosphere. The bottom left panel shows a series of solar flares, peaking in a relatively weak class M1 solar flare ( 3.2 times or $5 \mathrm{~dB}$ weaker than a rated "moderate" solar flare of class M5). Despite the relatively weak nature of the solar flare, the sferics are clearly perturbed. Most obviously higher frequency content is much stronger than both before and after the solar flare. Furthermore, the first rise in amplitude around $5 \mathrm{kHz}$ shifts, and the null starting around $30 \mathrm{kHz}$ for the pre-flare case shifts to much higher frequencies for the examples shown during the development of the solar flare. There are many other changes in the sferic characteristics which are very easily quantified
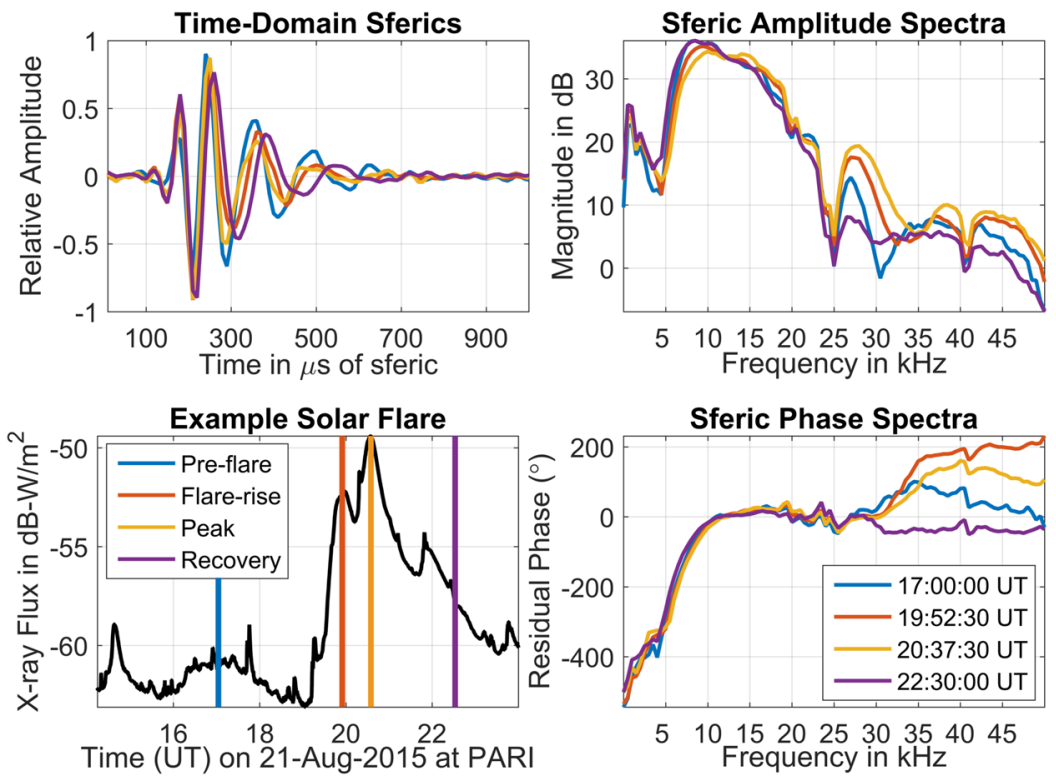

FIG. 14. Example of the effects of a solar flare on sferic propagation. The colored bars on the lower left figure indicate the time of the day and current solar x-ray flux in which example sferics were taken, and the color scheme is common to each panel. 


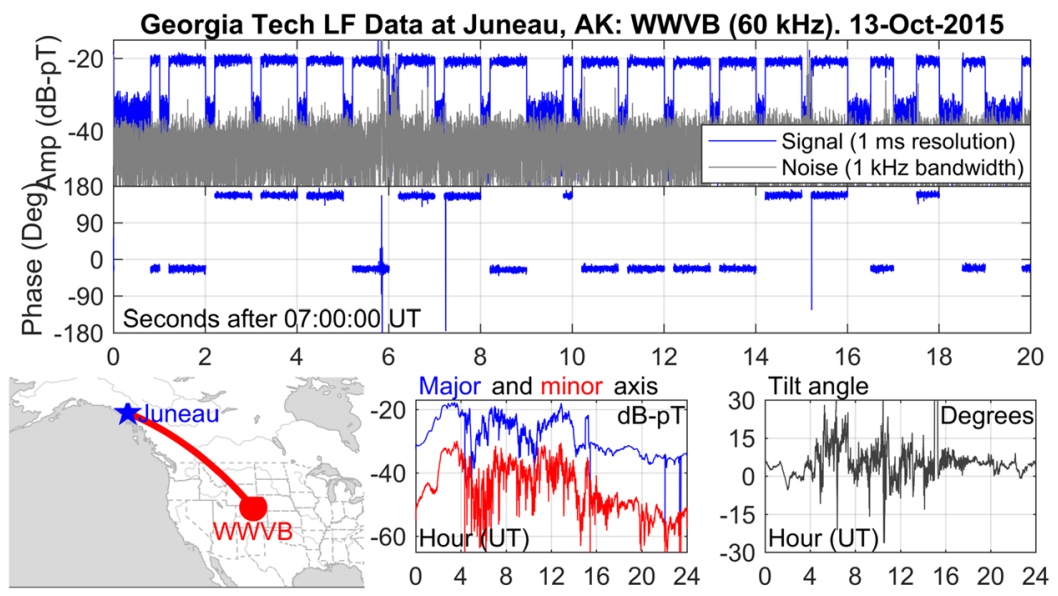

FIG. 15. WWVB Transmitter $(60 \mathrm{kHz})$ detected at a long range.

even for a relatively weak solar flare as in this example, and all of these perturbations are even more prominent for stronger sferics. A more complete description of this process is given in Ref. 150.

\section{LF/MF ionospheric remote sensing}

Figure 15 shows high-sensitivity detection of the WWVB $60 \mathrm{kHz}$ timing beacon operated by the National Institute of Standards and Technology (NIST) near Fort Collins, Colorado (40.67N, 105.04W), at $60 \mathrm{kHz}$. The NIST website states that the detection range for WWVB (defined as the radius where the signal strength is $100 \mu \mathrm{V} / \mathrm{m}$, or equivalently $0.33 \mathrm{pT}$ ) extends very close to Juneau at $7 \mathrm{UT}$, which is the maximum range extent during a typical day http://tf.nist.gov/stations/wwvbcoverage.htm. Figure 15 shows at the bottom left a map of WWVB and Juneau, where a receiver is located.

The previous AWESOME receiver cutoff was $47 \mathrm{kHz}$, so this transmitter was not recorded. With an increased sampling rate and sensitivity, the transmitter is detected at Juneau with a high SNR. The top panel of Fig. 15 shows in blue the amplitude of WWVB signal for a 20-s period and with $1 \mathrm{~ms}$ time resolution (or $1 \mathrm{kHz}$ of noise bandwidth). The signal has an amplitude-modulated code defined by the WWVB transmission format and a binary phase coding in addition. The gray trace shows the noise floor, which has a RMS amplitude of $\sim 3 \mathrm{fT}$, corresponding to a spectral noise of $\sim 0.1 \mathrm{fT} / \sqrt{\mathrm{Hz}}$. The center panel of Fig. 15 shows the phase as a function of time, which can be accurately calculated when the SNR is high. Like VLF transmitters, WWVB is a useful ionospheric remote sensing tool. The bottom center and bottom right panels show the amplitude and phase (modulo $180^{\circ}$ ), respectively, of the WWVB signal detected at Juneau. It is worth noting that the $15 \mathrm{~ns}$ accuracy of the sampling clock corresponds to a phase of $\sim 0.3^{\circ}$, whereas the phase appears to be jumping around over an $\sim 10^{\circ}$ range. This phase jumping is due to a weak SNR along with actual fast-scale ionospheric variation rather than timing jitter in the receiver.

The bottom center and bottom right panels of Fig. 15 show characteristics of the WWVB signal over a 24-h period on 13 August 2015. The amplitude and phase on the two horizontal components of the magnetic field are converted into a polarization ellipse, according to the techniques described by Ref. 136. The major axis length (blue trace) and minor axis length (red trace) are shown. The signal is highly linearly polarized for most of the day. The trace in the bottom right panel shows the tilt angle or the apparent arrival direction compared to the actual arrival direction from WWVB to Juneau. As the signal is largely dominated by TM modes, the arrival angle
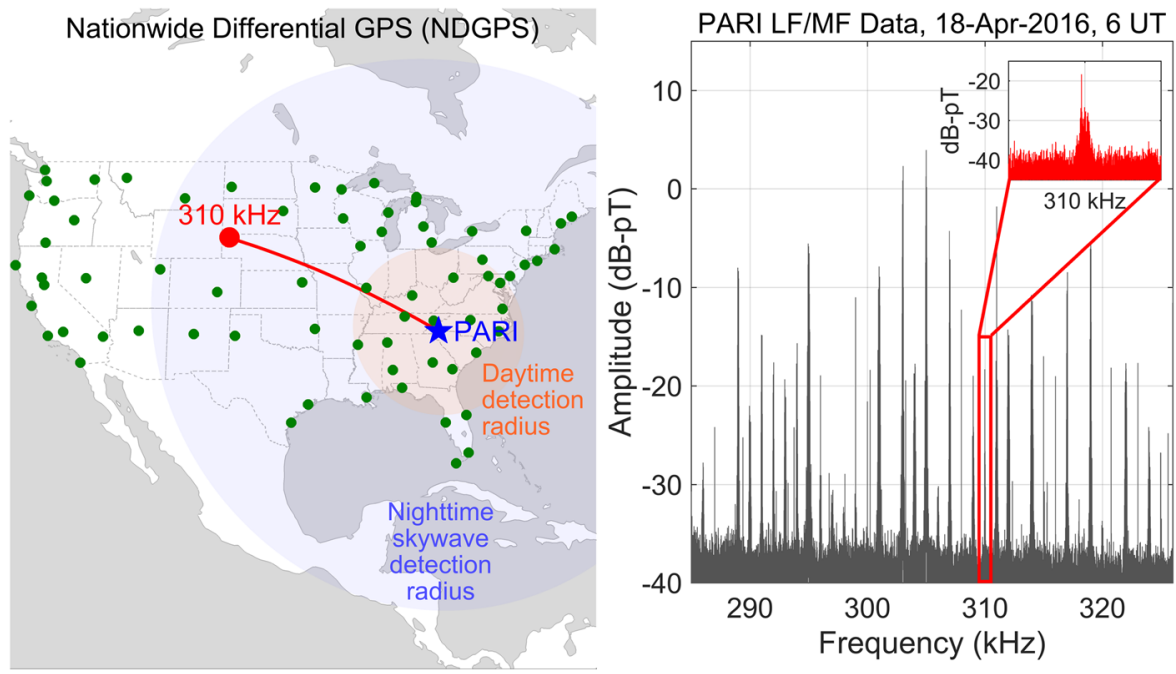

FIG. 16. NDGPS LF/MF beacons detected at a medium/long range. Adapted from Ref. 152. 
generally matches, although the effect of both TE modes and terrain features cannot be discounted. ${ }^{130,136,144,151}$

In addition, there are a number of radio beacons near $300 \mathrm{kHz}$ that can be detected at a long range. Figure 16 shows a constellation of transmitters that form the Nationwide Differential GPS (NDGPS) network, which is nominally used to broadcast ionospheric error corrections for more accurate GPS coverage and broadcast between 285 and $325 \mathrm{kHz}$. The left panel shows a map of the transmitters as they existed until July 2016, at which point about half of the 86 sites across the USA were shut down. On the right panel is an FFT of the entire spectrum in this range by the PARI receiver in North Carolina. The spectrum contains many signals from beacons up to $2500 \mathrm{~km}$ away. An example is shown via the $310 \mathrm{kHz}$ signal propagating from Nebraska, shown by a red trace on the map. The signal is detected at PARI with $10 \mathrm{~dB}$ SNR, as shown by the red thumbnail inside the right panel. So increasing the sensitivity and frequency range of the AWESOME opens up new avenues for ionospheric remote sensing in a frequency band that is not currently receiving much scientific focus. More details on LF/MF ionospheric remote sensing can be found in Ref. 152. A technique to combine the two horizontal channels into a polarization ellipse after MSK demodulation and its applications to ionospheric remote sensing can be found in Ref. 136. There have been some prior studies using LF/MF beacons for remote sensing of ionospheric disturbances. ${ }^{153}$

\section{CONCLUSION}

We have described an instrument capable of magnetic field detection in most of the ELF/VLF/LF frequency bands and extend into the MF band. The receiver builds on an earlier design but makes a number of important improvements.

We have shown the amplitude and phase response of the receiver, which includes the frequencies $500 \mathrm{~Hz}-470 \mathrm{kHz}$. We have characterized the noise levels and clipping threshold as a function of frequency, gain, and antenna size. We have characterized a custom-built timing adjustment algorithm that achieves $15 \mathrm{~ns}$ of absolute timing accuracy. We also described an MSK demodulation algorithm that deals with the multimode propagation nature of VLF and LF waves and can be used for ionospheric remote sensing.

Using this powerful hardware-software combination, we have described several useful applications to scientific remote sensing. We have demonstrated the demodulation of VLF transmitters at 20-30 kHz. We have shown lightning-generated radio atmospherics detected multiple times around the world due to efficient propagation and high sensitivity. We have shown that coherently averaged sferics are made possible in part by increased timing accuracy of the receiver. We have shown high-sensitivity detection of the amplitude and phase of the WWVB $60 \mathrm{kHz}$ transmitter and of NDGPS transmitters near $300 \mathrm{kHz}$.

\section{ACKNOWLEDGMENTS}

This work was supported by the National Science Foundation under Grant Nos. AGS 1451142 and 1653114 to the
Georgia Institute of Technology, by the Defense Advanced Research Project Agency under Grant No. HR0011-10-10058-P0001 to Stanford University with subcontract to Georgia Tech, and by a new faculty start-up grant from the College of Engineering at Georgia Tech. We thank our friends at the Pisgah Astronomical Research Institute for hosting a receiver.

${ }^{1}$ O. Bucci, G. Pelosi, and S. Selleri, IEEE Antennas Propag. Mag. 45, 46 (2003).

${ }^{2}$ E. V. Appleton, Nature 115, 382 (1925).

${ }^{3}$ E. Appleton, Inst. Electr. Eng.-Proc. Wireless Sect. Inst. 7, 257 (1932).

${ }^{4}$ R. Barr, D. L. Jones, and C. J. Rodger, J. Atmos. Sol.-Terr. Phys. 62, 1689 (2000).

${ }^{5}$ U. S. Inan, S. A. Cummer, and R. A. Marshall, J. Geophys. Res.: Space Phys. 115, A00E36, https://doi.org/10.1029/2009ja014775 (2010).

${ }^{6}$ I. Silber and C. Price, Surv. Geophys. 38, 407 (2017).

${ }^{7}$ G. J. Fishman, P. N. Bhat, R. Mallozzi, J. M. Horack, T. Koshut, C. Kouveliotou, G. N. Pendleton, C. A. Meegan, R. B. Wilson, and W. S. Paciesas, Science 264, 1313 (1994).

${ }^{8}$ V. A. Rakov and M. A. Uman, Lightning: Physics and Effects (Cambridge University Press, 2007).

${ }^{9}$ J. Galejs, Terrestrial Propagation of Long Electromagnetic Waves (Pergamon Press, 1972).

${ }^{10}$ C. Bruce and R. Golde, J. Inst. Electr. Eng.-Part II: Power Eng. 88, 487 (1941).

${ }^{11}$ R. K. Said, M. B. Cohen, and U. S. Inan, J. Geophys. Res.: Atmos. 118, 6905, https://doi.org/10.1002/jgrd.50508 (2013).

${ }^{12}$ H. J. Christian, R. J. Blakeslee, D. J. Boccippio, W. L. Boeck, D. E. Buechler, K. T. Driscoll, S. J. Goodman, J. M. Hall, W. J. Koshak, D. M. Mach, and M. F. Stewart, J. Geophys. Res. 108, 4005, https://doi.org/10.1029/2002jd002347 (2003).

${ }^{13}$ C. Barrington-Leigh and U. S. Inan, Geophys. Res. Lett. 26, 683, https://doi.org/10.1029/1999g1900059 (1999).

${ }^{14}$ C. T. R. Wilson, Proc. Phys. Soc. London 37, 32D (1925).

${ }^{15}$ R. C. Franz, R. J. Nemzek, and J. R. Winckler, Science 249, 48 (1990)

${ }^{16}$ T. Neubert, T. H. Allin, H. Stenbaek-Nielsen, and E. Blanc, Geophys. Res. Lett. 28, 3585, https://doi.org/10.1029/2001g1013427 (2001).

${ }^{17}$ M. Hayakawa, T. Nakamura, Y. Hobara, and E. Williams, J. Geophys. Res. 109, A01312, https://doi.org/10.1029/2003ja009905 (2004).

${ }^{18} \mathrm{M}$. Ignaccolo, T. Farges, A. Mika, T. H. Allin, O. Chanrion, E. Blanc, T. Neubert, A. C. Fraser-Smith, and M. Füllekrug, Geophys. Res. Lett. 33, L11808, https://doi.org/10.1029/2005gl025502 (2006).

${ }^{19}$ Á. Mika and C. Haldoupis, Space Sciences Series of ISSI (Springer, New York, 2008), pp. 489-510.

${ }^{20}$ M. J. Taylor, M. A. Bailey, P. D. Pautet, S. A. Cummer, N. Jaugey, J. N. Thomas, N. N. Solorzano, F. S. Sabbas, R. H. Holzworth, O. Pinto, and N. J. Schuch, Geophys. Res. Lett. 35, L14812, https://doi.org/10.1029/2008g1033984 (2008).

${ }^{21}$ S. NaitAmor, M. A. AlAbdoadaim, M. B. Cohen, B. R. T. Cotts, S. Soula, O. Chanrion, O. Neubert, and T. Abdelatif, J. Geophys. Res.: Space Phys. 115, A00E47, https://doi.org/10.1029/2009JA015026 (2010).

${ }^{22}$ S. NaitAmor, M. B. Cohen, B. R. T. Cotts, H. Ghalila, M. A. Alabdoadaim, and K. L. Graf, J. Geophys. Res.: Space Phys. 118, 5215, https://doi.org/10.1002/jgra.50448 (2013).

${ }^{23}$ H. T. Su, Nature 423, 974 (2003).

${ }^{24}$ V. P. Pasko, M. A. Stanley, J. D. Mathews, U. S. Inan, and T. G. Wood, Nature 416, 152 (2002).

${ }^{25}$ M. Parrot, U. S. Inan, and N. G. Lehtinen, J. Geophys. Res.: Space Phys. 113, A10310, https://doi.org/10.1029/2008JA013336 (2008).

${ }^{26}$ W. C. Armstrong, Antarct. J. 18, 281 (1983).

${ }^{27}$ W. B. Peter and U. S. Inan, J. Geophys. Res.: Space Phys. 112, A12212, https://doi.org/10.1029/2006JA012165 (2007).

${ }^{28}$ E. R. Swanson, Proc. IEEE 71, 1140 (1983).

${ }^{29}$ A. D. Watt, VLF Radio Engineering (Pergamon Press, 1967).

${ }^{30}$ R. Raghuram, R. L. Smith, and T. F. Bell, IEEE Trans. Antennas Propag. 22, 334 (1974).

${ }^{31}$ H. Wheeler, IRE Trans. Antennas Propag. 6, 123 (1958).

${ }^{32}$ N. R. Thomson, J. Atmos. Terr. Phys. 55, 173 (1993).

${ }^{33}$ N. R. Thomson, M. A. Clilverd, and W. M. McRae, J. Geophys. Res.: Space Phys. 112, A07304, https://doi.org/10.1029/2007JA012271 (2007).

${ }^{34}$ C. F. J. Sechrist, Radio Sci. 9, 137, https://doi.org/10.1029/rs009i002p00137 (1974). 
${ }^{35}$ C. G. Getmantsev, N. A. Zuikov, D. S. Kotik, N. A. Mironenko, V. O. Mityakov, Y. A. Rapoport, V. Y. Sazanov, V. Y. Trakhtengerts, and V. Y. Eidman, JETP Lett. 20, 101 (1974).

${ }^{36}$ P. Stubbe, H. Kopka, and R. L. Dowden, J. Geophys. Res. 86, 9073, https://doi.org/10.1029/ja086ia11p09073 (1981).

${ }^{37}$ R. C. Moore, U. S. Inan, T. F. Bell, and E. J. Kennedy, J. Geophys. Res.: Space Phys. 112, A05309, https://doi.org/10.1029/2006JA012063 (2007).

${ }^{38}$ M. Golkowski, M. B. Cohen, D. L. Carpenter, and U. S. Inan, J. Geophys. Res.: Space Phys. 116, A04208, https://doi.org/10.1029/2010ja016261 (2011).

${ }^{39}$ M. B. Cohen and M. Golkowski, J. Geophys. Res.: Space Phys. 118, 6597, https://doi.org/10.1002/jgra.50558 (2013).

${ }^{40}$ S. Ananthakrishnan, M. Abdu, and L. Piazza, Planet. Space Sci. 21, 367 (1973).

${ }^{41}$ A. P. Mitra, Ionospheric Effects of Solar Flares (Reidel, Dordrecht, The Netherlands, 1974).

${ }^{42}$ W. C. Bain and E. Hammond, J. Atmos. Terr. Phys. 37, 573 (1975).

${ }^{43}$ N. R. Thomson and M. A. Clilverd, J. Atmos. Sol.-Terr. Phys. 63, 1729 (2001).

${ }^{44}$ N. R. Thomson, J. Geophys. Res. 110, A06306, https://doi.org/10.1029/ 2005ja011008 (2005).

${ }^{45}$ V. Žigman, D. Grubor, and D. Šulić, J. Atmos. Sol.-Terr. Phys. 69, 775 (2007).

${ }^{46}$ D. Šulić, V. Srećković, and A. Mihajlov, Adv. Space Res. 57, 1029 (2016).

${ }^{47}$ M. A. Clilverd, C. J. Rodger, T. Ulich, A. Seppälä, E. Turunen, A. Botman, and N. R. Thomson, J. Geophys. Res.: Space Phys. 110, A09307, https://doi.org/10.1029/2004ja010922 (2005).

${ }^{48}$ M. A. Clilverd, A. Seppälä, C. J. Rodger, N. R. Thomson, P. T. Verronen, E. Turunen, T. Ulich, J. Lichtenberger, and P. Steinbach, Radio Sci. 41, RS2001, https://doi.org/10.1029/2005rs003290 (2006).

${ }^{49}$ E. B. Burton and E. M. Boardman, Nature 131, 81 (1933).

${ }^{50}$ P. Kaufmann and R. E. Schaal, J. Atmos. Terr. Phys. 30, 469 (1968).

${ }^{51}$ R. F. Hoy, J. Atmos. Terr. Phys. 31, 1027 (1969).

${ }^{52}$ R. J. Doherty, Nature 226, 1129 (1970).

${ }^{53}$ K. Lynn, "The total solar eclipse of 23 October 1976 observed at VLF," Technical Report ERL-0093-TR, Defense Science and Technology Organization, 1981.

${ }^{54}$ K. Cheng, Y.-N. Huang, and S.-W. Chen, J. Geophys. Res. 97, 103, https://doi.org/10.1029/91ja02409 (1992).

${ }^{55}$ M. A. Clilverd, C. J. Rodger, N. R. Thomson, J. Lichtenberger, P. Steinback, P. Cannon, and M. J. Angling, Radio Sci. 36, 773, https://doi.org/10.1029/2000rs002395 (2001).

${ }^{56}$ V. I. Kozlov, R. R. Karimov, and V. A. Mullayarov, Russ. Phys. J. 50, 617 (2007).

${ }^{57}$ L. F. Chernogor, Geomagn. Aeron. 50, 96 (2010).

${ }^{58}$ R. Singh, B. Veenadhari, A. K. Maurya, M. B. Cohen, S. Kumar, R. Selvakumaran, P. Pant, A. K. Singh, and U. S. Inan, J. Geophys. Res.: Space Phys. 116, A10301, https://doi.org/10.1029/2011JA016641 (2011).

${ }^{59}$ M. B. Cohen, N. C. Gross, M. A. Higginson-Rollins, R. A. Marshall, M. Gołkowski, W. Liles, D. Rodriguez, and J. Rockway, Geophys. Res. Lett. 45, 3348, https://doi.org/10.1002/2018g1077351 (2018).

${ }^{60}$ N. R. Thomson, M. A. Clilverd, and C. J. Rodger, J. Geophys. Res.: Space Phys. 119, 6865, https://doi.org/10.1002/2014ja020299 (2014).

${ }^{61}$ G. J. Fishman and U. S. Inan, Nature 331, 418 (1988).

${ }^{62}$ U. S. Inan, N. G. Lehtinen, S. J. Lev-Tov, M. P. Johnson, T. F. Bell, and K. Hurley, Geophys. Res. Lett. 26, 3357, https://doi.org/ 10.1029/1999g1010690 (1999).

${ }^{63}$ U. S. Inan, N. G. Lehtinen, R. C. Moore, K. Hurley, S. Boggs, D. M. Smith, and G. J. Fishman, Geophys. Res. Lett. 34, L08103, https://doi.org/10.1029/2006GL029145 (2007).

${ }^{64}$ Y. T. Tanaka, J.-P. Raulin, F. C. P. Bertoni, P. R. Fagundes, J. Chau, N. J. Schuch, M. Hayakawa, Y. Hobara, T. Terasawa, and T. Takahashi, Astrophys. J. 721, L24 (2010).

${ }^{65}$ M. S. Solovieva and A. A. Rozhnoi, Geomagn. Aeron. 55, 805 (2015)

${ }^{66}$ P. A. Kossey, J. P. Turtle, R. P. Pagliarulo, W. I. Klemetti, and J. E. Rasmussen, Radio Sci. 18, 907, https://doi.org/10.1029/rs018i006p00907 (1983).

${ }^{67}$ S. A. Cummer, T. F. Bell, U. S. Inan, and D. L. Chenette, J. Geophys. Res.: Space Phys. 102, 7477, https://doi.org/10.1029/96ja03721 (1997).

${ }^{68}$ J. S. Belrose and L. Thomas, J. Atmos. Terr. Phys. 30, 1397 (1968).

${ }^{69}$ C. J. Rodger, M. A. Clilverd, N. R. Thomson, R. J. Gamble, A. Sappala, E. Turunen, N. P. Meredith, M. Parrot, J. A. Sauvaud, and J. J. Berthelier, J. Geophys. Res.: Space Phys. 112, A11307, https://doi.org/10.1029/2007JA012383 (2007).

${ }^{70}$ M. A. Abdu, I. S. Batista, L. R. Piazza, and O. Massambani, J. Geophys. Res.: Space Phys. 86, 7533, https://doi.org/10.1029/ja086ia09p07533 (1981).

${ }^{71}$ O. Pinto, W. Gonzalez, and N. Leme, Planet. Space Sci. 38, 633 (1990).

${ }^{72}$ R. C. Moore, C. P. Barrington-Leigh, U. S. Inan, and T. F. Bell, J. Geophys. Res. 108, 1363, https://doi.org/10.1029/2002JA009816 (2003).

${ }^{73}$ C. Haldoupis, T. Neubert, U. S. Inan, A. Mika, T. H. Allin, and R. A. Marshall, J. Geophys. Res. 109, A10303, https://doi.org/ 10.1029/2004JA010651 (2004).

${ }^{74}$ M. C. Kelley, C. L. Siefring, R. F. Pfaff, P. M. Kintner, M. Larsen, R. Green, R. H. Holzworth, L. C. Hale, J. D. Mitchell, and D. Le Vine, J. Geophys. Res. 90, 9815, https://doi.org/10.1029/ja090ia10p09815 (1985).

${ }^{75}$ R. A. Marshall and U. S. Inan, J. Geophys. Res.: Space Phys. 115, A00E29, https://doi.org/10.1029/2009JA014761 (2010).

${ }^{76}$ C. Haldoupis, M. B. Cohen, R. Arnone, B. R. T. Cotts, and S. Dietrich, J. Geophys. Res.: Space Phys. 118, 5392, https://doi.org/10.1002/jgra.50489 (2013).

${ }^{77}$ U. S. Inan, Geophys. Res. Lett. 17, 729, https://doi.org/10.1029/ g1017i006p00729 (1990).

${ }^{78}$ K. L. Graf, U. S. Inan, and M. Spasojevic, J. Geophys. Res.: Space Phys. 116, A12313, https://doi.org/10.1029/2011JA016996 (2011).

${ }^{79}$ M. A. Clilverd, J. Geophys. Res. 109, A12208, https://doi.org/10.1029/ 2004ja010644 (2004)

${ }^{80}$ J. Bortnik, U. S. Inan, and T. F. Bell, J. Geophys. Res 111, A02204, https://doi.org/10.1029/2005ja011398 (2006).

${ }^{81}$ U. S. Inan, M. Golkowski, M. K. Casey, R. C. Moore, W. Peter, P. Kulkarni, P. Kossey, E. Kennedy, S. Z. Meth, and P. Smit, Geophys. Res. Lett. 34, L02106, https://doi.org/10.1029/2006GL028494 (2007).

${ }^{82}$ R. J. Gamble, C. J. Rodger, M. A. Clilverd, J.-A. Sauvaud, N. R. Thomson, S. L. Stewart, R. J. McCormick, M. Parrot, and J.-J. Berthelier, J. Geophys. Res.: Space Phys. 113, A10211, https://doi.org/10.1029/2008ja013369 (2008).

${ }^{83}$ D. Cavalieri, R. Deland, T. Potemra, and R. Gavin, J. Atmos. Terr. Phys. 36, 561 (1974).

${ }^{84}$ E. D. Schmitter, Ann. Geophys. 29, 1287 (2011).

${ }^{85}$ A. Nina and V. M. Cadez, Geophys. Res. Lett. 40, 4803, https://doi.org/10.1002/grl.50931 (2013).

${ }^{86}$ R. A. Marshall and J. B. Snively, J. Geophys. Res.: Atmos. 119, 5037, https://doi.org/10.1002/2014jd021594 (2013).

${ }^{87}$ M. B. Goghberg, V. A. Morgounov, T. Yoshino, and I. Tomizawa, J. Geophys. Res. 87, 7824, https://doi.org/10.1029/jb087ib09p07824 (1982).

${ }^{88}$ M. A. Clilverd, C. J. Rodger, and N. R. Thomson, J. Geophys. Res. 104, 28171, https://doi.org/10.1029/1999ja900285 (1999).

${ }^{89}$ F. Nemec, O. Santolik, and M. Parrot, J. Geophys. Res.: Space Phys. 114, A04303, https://doi.org/10.1029/2008JA013972 (2009).

${ }^{90}$ M. B. Cohen and R. A. Marshall, Geophys. Res. Lett. 39, L11804, https://doi.org/10.1029/2012g1052123 (2012)

${ }^{91}$ I. Silber, R. Price, C. Rodger, and C. Haldoupis, J. Geophys. Res.: Atmos. 118, 4244, https://doi.org/10.1002/jgrd.50379 (2013).

${ }^{92}$ M. E. Andersson, P. T. Verronen, S. Wang, C. J. Rodger, M. A. Clilverd, and B. R. Carson, J. Geophys. Res.: Atmos. 117, D09304, https://doi.org/10.1029/2011jd017246 (2012).

${ }^{93}$ O. I. Pintado, S. M. Radicella, and P. M. Fernández, J. Atmos. Terr. Phys. 49, 129 (1987).

${ }^{94}$ A. Bailey and H. M. Thomson, Bell Syst. Tech. J. 14, 680 (1935).

${ }^{95}$ A. A. Pacini and J.-P. Raulin, J. Geophys. Res. 111, A09301, https://doi.org/10.1029/2006ja011613 (2006).

${ }^{96}$ A. Rozhnoi, M. Solovieva, B. Levin, M. Hayakawa, and V. Fedun, Nat. Hazards Earth Syst. Sci. 14, 2671 (2014).

${ }^{97}$ A. H. Brady and D. D. Crombie, J. Geophys. Res. 68, 5437, https://doi.org/10.1029/jz068i019p05437 (1963).

${ }^{98}$ F. C. P. Bertoni, J.-P. Raulin, H. R. Gavilán, P. Kaufmann, R. Rodriguez, M. Clilverd, J. S. Cardenas, and G. Fernandez, J. Geophys. Res.: Space Phys. 118, 6686, https://doi.org/10.1002/jgra.50559 (2013).

${ }^{99}$ R. A. Helliwell, Adv. Space Res. 8, 279 (1988).

${ }^{100}$ M. B. Cohen and U. S. Inan, J. Geophys. Res.: Space Phys. 117, A08310, https://doi.org/10.1029/2012JA017992 (2012).

${ }^{101}$ M. B. Cohen, N. G. Lehtinen, and U. S. Inan, Geophys. Res. Lett. 39, L24101, https://doi.org/10.1029/2012GL054437 (2012).

${ }^{102}$ B. Abel and R. M. Thorne, J. Geophys. Res.: Space Phys. 103, 2385, https://doi.org/10.1029/97ja02919 (1998).

${ }^{103}$ E. B. Burton, Nature 126, 55 (1930). 
${ }^{104}$ M. Yamashita, J. Atmos. Terr. Phys. 40, 151 (1978).

${ }^{105}$ A. G. Jean, W. L. Taylor, and J. R. Wait, J. Geophys. Res. 65, 907, https://doi.org/10.1029/jz065i003p00907 (1960).

${ }^{106}$ V. A. Rafalsky, A. V. Shvets, and M. Hayakawa, J. Atmos. Sol.-Terr. Phys. 57, 1255 (1995).

${ }^{107}$ S. A. Cummer, U. S. Inan, and T. Bell, Radio Sci. 33, 1781, https://doi.org/10.1029/98rs02381 (1998).

${ }^{108}$ E. H. Lay and X. M. Shao, J. Geophys. Res.: Space Phys. 116, A01317, https://doi.org/10.1029/2010JA016018 (2011).

${ }^{109}$ X. M. Shao, E. H. Lay, and A. R. Jacobson, Nat. Geosci. 7, 29 (2013).

${ }^{110}$ United States Coast Guard, "LORAN-C user's handbook," Technical Report COMDTINST M16562.3, United States Department of Transportation, 1980.

${ }^{111}$ M. Füllekrug, Meas. Sci. Technol. 21, 015901 (2009).

${ }^{112}$ A. C. Fraser-Smith and R. A. Helliwell, in Proceedings of IEEE International Symposium on Electromagnetic Compatibility (IEEE, 1985).

${ }^{113}$ H. C. Séran and P. Fergeau, Rev. Sci. Instrum. 76, 044502 (2005).

${ }^{114}$ M. Parrot, D. Benoist, J. J. Berthelier, J. Blecki, Y. Chapuis, F. Colin, F. Elie, P. Fergeau, D. Lagoutte, F. Lefeuvre, C. Legendre, M. Leveque, J. L. Pincon, B. Poirier, H.-C. Seran, and P. Zamora, Planet. Space Sci. 54, 441 (2006).

${ }^{115}$ C. Cavoit, Rev. Sci. Instrum. 77, 064703 (2006).

${ }^{116} \mathrm{~T}$. Bolton and M. B. Cohen, "Optimal design of electrically-small loop receiving antenna," IEEE Trans. Antennas Propag. (submitted).

${ }^{117}$ J. P. Wikswo, P. C. Samson, and R. P. Giffard, IEEE Trans. Biomed. Eng. BME-30, 215 (1983).

${ }^{118}$ T. Eriksson, J. Blomgren, D. Winkler, T. Holst, and Y. Q. Shen, IEEE Trans. Appl. Supercond. 9, 3495 (1999).

${ }^{119}$ J. Lepaisant, M. L. C. Sing, and D. Bloyet, Rev. Sci. Instrum. 63, 2089 (1992).

${ }^{120}$ R. Sklyar, IEEE Sens. J. 5, 924 (2005).

${ }^{121}$ V. Gerginov, F. C. S. da Silva, and D. Howe, Rev. Sci. Instrum. 88, 125005 (2017).

${ }^{122}$ E. W. Paschal, "The design of broad-band VLF receivers with air-core loop antennas," Technical Report, STARLab, Stanford University, 1980.

${ }^{123}$ S. K. Harriman, E. W. Paschal, and U. S. Inan, IEEE Trans. Geosci. Remote Sens. 48, 396 (2010).

${ }^{124}$ M. B. Cohen, U. S. Inan, and E. P. Paschal, IEEE Trans. Geosci. Remote Sens. 48, 3 (2010).

${ }^{125}$ D. Scherrer, M. Cohen, T. Hoeksema, U. Inan, R. Mitchell, and P. Scherrer, Adv. Space Res. 42, 1777 (2008).

${ }^{126}$ B. J. Thompson, N. Gopalswamy, J. M. Davila, and H. J. Haubold, Putting the "I" in IHY (SpringerWien, 2009).

${ }^{127}$ R. K. Said, U. S. Inan, and K. L. Cummins, J. Geophys. Res. 115, D23108, https://doi.org/10.1029/2010JD013863 (2010).

${ }^{128}$ I. Silber, C. Price, E. Galanti, and A. Shuval, J. Geophys. Res.: Space Phys. 120, 6036, https://doi.org/10.1002/2015JA021141 (2015).

${ }^{129}$ U. S. Inan, M. Gołkowski, D. L. Carpenter, N. Reddell, R. C. Moore, T. F. Bell, E. W. Paschal, P. Kossey, E. Kennedy, and S. Z. Meth, Geophys. Res. Lett. 31, L24805, https://doi.org/10.1029/2004GL021647 (2004).

${ }^{130}$ F. G. Zoghzoghy, M. B. Cohen, R. K. Said, N. G. Lehtinen, and U. S. Inan, J. Geophys. Res.: Atmos. 120, 10890, https://doi.org/10.1002/2015jd023226 (2015).
${ }^{131}$ M. A. Stuchly, H. LePoncher, D. T. Gibbons, and A. Thansadote, IEEE Trans. Electromagn. Compat. 33, 275 (1991).

${ }^{132}$ A. C. Fraser-Smith, A. Bernardi, P. R. McGill, M. E. Ladd, R. A. Helliwell, and O. G. Villard, Jr., Geophys. Res. Lett. 17, 1465 , https://doi.org/10.1029/g1017i009p01465 (1990).

${ }^{133}$ D. A. Chrissan and A. C. Fraser-Smith, Radio Sci. 31, 1141, https://doi.org/10.1029/96rs01930 (1996).

${ }^{134}$ D. A. Chrissan and A. C. Fraser-Smith, Radio Sci. 38, 1078 , https://doi.org/10.1029/2002RS002693 (2003).

${ }^{135}$ M. B. Cohen, R. K. Said, and U. S. Inan, Radio Sci. 45, R6002, https://doi.org/10.1029/2010RS004420 (2010).

${ }^{136}$ N. G. Gross, M. B. Cohen, R. K. Said, and M. A. Golkowski, J. Geophys. Res.: Space Phys. 123, 901, https://doi.org/10.1002/2017ja024907 (2018).

${ }^{137}$ W. P. Osborne and M. B. Luntz, IEEE Trans. Commun. 22, 1023 (1974).

${ }^{138}$ M. S. El-Tanany and S. A. Mahmoud, IEEE J. Sel. Areas Commun. 5, 896 (1987).

${ }^{139}$ M. Morelli and G. M. Vitetta, IEEE Trans. Commun. 48, 1997 (2000).

${ }^{140}$ M. Füllekrug, A. Mezentsev, R. Watson, S. Gaffet, I. Astin, and A. Evans, Geophys. Res. Lett. 41, 9143, https://doi.org/10.1002/2014g1062126 (2014).

${ }^{141}$ M. Füllekrug, N. Smith, A. Mezentsev, R. Watson, I. Astin, S. Gaffet, A. Evans, and M. Rycroft, Radio Sci. 50, 1141, https://doi.org/10.1002/2015rs005781 (2015).

${ }^{142}$ R. L. Dowden and C. D. D. Adams, J. Geophys. Res. 94, 1515, https://doi.org/10.1029/ja094ia02p01515 (1989).

${ }^{143}$ C. D. D. Adams and R. L. Dowden, J. Geophys. Res.: Space Phys. 95, 2457, https://doi.org/10.1029/ja095ia03p02457 (1990).

${ }^{144}$ R. K. Said, "Accurate and efficient long-range lightning geo-location using a VLF radio atmospheric waveform bank," Ph.D. thesis, Stanford University, 2009.

${ }^{145}$ M. J. Rycroft, S. Israelsson, and C. Price, J. Atmos. Sol. Terr. Phys. 62, 1563 (2000).

${ }^{146}$ B. P. Besser, Radio Sci. 42, RS2S02, https://doi.org/10.1029/2006rs003495 (2007).

${ }^{147}$ M. Ishaq and D. L. Jones, Electron. Lett. 13, 254 (1977).

${ }^{148}$ T. Ogawa, Y. Tanaka, M. Miura, and T. Yasuhara, J. Geomagn. Geoelectr. 18, 443-454 (1966).

${ }^{149}$ T. Ogawa, Y. Tanaka, M. Yasuhara, A. C. Fraser-Smith, and R. Gendrin, J. Geomagn. Geoelectr. 19, 377 (1967).

${ }^{150}$ J. C. McCormick, M. B. Cohen, N. G. Gross, and R. K. Said, J. Geophys. Res.: Space Phys. 123, 3111, https://doi.org/10.1002/2017ja024291 (2018).

${ }^{151}$ T. G. Wood and U. S. Inan, J. Geophys. Res. 107, 4553, https://doi.org/ 10.1029/2001jd000659 (2002).

${ }^{152}$ M. A. Higginson-Rollins and M. B. Cohen, Geophys. Res. Lett. 44, 8665, https://doi.org/10.1002/2017gl074236 (2017).

${ }^{153}$ D. L. Carpenter, U. S. Inan, M. L. Trimpi, R. A. Helliwell, and J. P. Katsufrakis, J. Geophys. Res. 89, 9857, https://doi.org/ 10.1029/ja089ia11p09857 (1984). 